\title{
Nominal Uniqueness and Money Non-neutrality in the Limit-Price Exchange Process
}

\author{
Gä̈L GiRAud sj* \\ CNRS, Paris School of Economics, CERAS, France. \\ $\&$ \\ Dimitrios P. Tsomocos ${ }^{\dagger}$ \\ Saïd Business School University \\ St. Edmund Hall, University of Oxford \& Financial Markets Group, L. S. E., UK. ${ }^{\ddagger}$
}

June 7, 2010

\begin{abstract}
We define continuous-time dynamics for exchange economies with fiat money. Traders have locally rational expectations, face a cash-in-advance constraint, and continuously adjust their short-run dominant strategy in a monetary strategic market game involving a double-auction with limit-price orders. Money has a positive value except on optimal rest-points where it becomes a "veil" and trade vanishes. Typically, there is a piecewise globally unique trade-and-price curve both in real and in nominal variables. Money is not neutral, either in the short-run or long-run, and a localized version of the quantity theory of money holds in the short-run. An optimal money growth rate is derived, which enables monetary trade curves to converge towards Pareto optimal rest-points. Below this growth rate, the economy enters a (sub-optimal) liquidity trap where monetary policy is ineffective; above this threshold inflation rises. Finally, market liquidity, measured through the speed of real trades, can be linked to gains-to-trade, households' expectations, and the quantity of circulating money.
\end{abstract}

Keywords. Bank; Money; Price-quantity Dynamics; Inside money; Outside money; Rational expectations; Liquidity; Double auction; Limit-price Orders ; Inflation ; Bounded rationality.

JEL Classification: D50, D83, E12, E24, E30, E40, E41, E50, E58.

*ggiraud@parisschoolofeconomics.eu

${ }^{\dagger}$ dimitrios.tsomocos@said-business-school.oxford.ac.uk

${ }^{\ddagger}$ We are grateful to seminar participants at the 1st Annual CARESS-Cowles Conference on General Equilibrium and its Applications, New Haven, the General Equilibrium Workshop, Zürich, the University of Strasburg and Paris-1, and especially J.-M. Bonnisseau, B. Cornet, J. Geanakoplos, A. Mas-Colell and H. Polemarchakis for helpful comments. All remaining errors are ours. An earlier version of this paper was circulated under a different title, see Giraud \& Tsomocos (2004). 


\section{Introduction}

Most macroeconomic models reduce the aggregate economy to manageable proportions, and usually a common simplification is the representation of each sector by agents which behave identically. Consequently, they are presented in "representative agent" format. On the other hand, standard general equilibrium with heterogenous agents quickly becomes intractable. Closed form solutions cannot be derived and their results are often not robust. The main impediment lies on the multiplicity of equilibria. A second drawback is that the economy is assumed to be always at equilibrium while the theory is unable to describe in a sensible way what happens out of equilibrium.

This paper extends the monetary paradigm set out in Dubey \& Geanakoplos (1992) and earlier working papers by providing a microfounded solution for the price discovery process via a series of intermediate trades and establishes global uniqueness of the solution paths. In doing so, we use novel but intuitive techniques that can be used in subsequent research using the Mertens (2003) limit-price mechanism. The emergence of the monetary gains-to-trade hypothesis to explain the speed and volume of trades provides a direct rôle for monetary policy in affecting market transactions and results in a new interpretation for the quantity theory of money (QTM). While maintaining market clearing and agent optimization, we follow the spirit (originating in Shubik \& Wilson (1977)) of introducing money in general equilibrium theory via a Central Bank. At each intermediate trade, agents can borrow money from a Central Bank and take part to a monetary double auction on the commodity market, sending limit-price orders to a clearing house. However, being boundedly rational, households cannot solve the intertemporal optimization programme of their future discounted utility: Given individual expectations, they try to move in the direction of the steepest increase of their current utility. $^{1}$

Given the myopic behavior of households postulated in this paper, Lucas's concept of rational expectations needs to be adapted to our context. On the other hand, we have learned from temporary equilibrium theory, ${ }^{2}$ that allowing for arbitrary "animal spirits" (in the sense of Keynes) can prevent from getting locally unique equilibria. Here, the main impact of expectations lies on the amount of money and commodities spent on markets and, correspondingly, on savings. We shall assume that investors form locally rational forecasts, and behave accordingly - a forecast being "locally rational" whenever it is self-fulfilling in the short-run. This way of capturing households' expectations results into far-reaching consequences regarding the characterization of solution path of our dynamics.

The striking feature of our model, indeed, is that it fully describes out-of-equilibrium

\footnotetext{
${ }^{1}$ The economic rationale for such myopic behavior can be traced back at least to Smale's work (1976a,b, 1977). As for its plausibility, recall that even chess grandmasters do not calculate more than four or five moves ahead. It has also been suggested that, in situations such as chess, seeing further does not mean seeing better (Gray \& Geanakoplos (1991)). Finally, experience from financial market desks shows that striving for the "local steepest increase" of one's current P \& L is indeed close to most traders' daily behavior.

${ }^{2}$ See Grandmont (2007) and the references therein.
} 
behaviour but, more importantly, it also possesses globally unique solution paths. Unlike most, if not all, of OLG and many General Equilibrium models that exhibit huge degrees of indeterminacy, our framework produces globally unique trade paths solving our dynamics. Thus, our approach is capable of providing an integrated framework to conduct macroeconomic analysis and comparative dynamics without resorting to the representative agent artifact with its well-established shortcomings. ${ }^{3}$ On the doctrinal level, our approach also provides a new "New Classical synthesis" between Keynesian wisdom (money is non-neutral, liquidity trap may occur) and the monetarist viewpoint (QTM holds under certain circumstances). ${ }^{4}$ To the best of our knowledge, this is the first attempt to combine all these features together: An Arrow-Debreu setting of out-ofequilibrium behavior founded on a full-blown game-theoretic microstructure with nonneutral money, global uniqueness of tractable solutions and some version of QTM. ${ }^{5}$

\subsection{Gains-to-trade, expectations and money}

The dynamics is driven, at each time instant, by a "tangent market" where agents trade infinitesimal amounts of goods and maximize the first-order approximation of their current utilities subject to a cash-in-advance constraint à la Clower (1967). ${ }^{6}$ Hence, our approach can be viewed as the monetary counterpart of the limit-price exchange process introduced by Giraud (2004), which is itself a game-theoretic rewriting of Champsaur \& Cornet (1990).

Receipts from commodity sales cannot be used contemporaneously for purchases. Therefore, in order to fulfill their cash-in-advance constraint, traders borrow money from a loan market in anticipation of future income which is used to defray their loans. Agents are endowed both with commodities as well as with some cash which is owned free and clear of any debt. The aggregate of all private monetary holdings is the outside money. The Central Bank loans money to agents who, in turn, repay after they have received income from the sales of their commodities. Hence, for Central Bank issued money, there exists an offsetting liability, which induces its exit from the economy. This money is called inside money. ${ }^{7}$

A short-run interest rate is endogenously determined at each instant and clears the money market. We exhibit necessary and sufficicient conditions under which the "classical dichotomy" holds in the short-run in the form of Fisher's quantity theory of money, provided there are effective trades. In particular, our localized version of the quantity theory of money depends upon a "gains-to-trade" hypothesis first introduced by Dubey \& Geanakoplos (1992, 2003a, 2006). Consequently, when these conditions are fulfilled, in the short-run, one can separate the real and nominal sides of the economy, solving

\footnotetext{
${ }^{3}$ Cf. e.g., Kirman (1992).

${ }^{4}$ See Giraud (2009) for a discussion of the present model within the history of dynamics in General Equilibrum Theory (GET).

${ }^{5}$ In addition, our dynamics is computable. The numerical aspects will be explored in a companion paper.

${ }^{6}$ See also Grandmont \& Younès (1972).

${ }^{7}$ The distinction between outside and inside money has been introduced by Gurley \& Shaw (1960).
} 
the real side for relative prices, and fixing their levels by the stock of nominal money. But this holds only in the short-run because the ratio of outside to inside money must change over time in order to compensate for the diminution of gains to trade. Otherwise, trades collapse, and the state of the economy remains stuck in a "liquidity trap" at some (possibly second-best) commodity allocation. How the amount of inside money changes, necessarily affects both nominal and real variables along every trade curve. Finally, not only does money have value in our model, ${ }^{8}$ but its value is determinate. For generic economies, the dynamics of interest rates, price levels and commodity allocations is shown to be piecewise unique in forward time so that the non-neutral long-run effects of monetary policy can in principle be tracked. ${ }^{9}$

Every Pareto-optimal price equilibrium is a rest-point of the dynamics. However, as it is to be expected from a non-tâtonnement approach, ${ }^{10}$ and even if there is no money, there is no reason, in general, for such a rest-point to be an equilibrium of the economy starting at some other state along the trade-curve. Thus, even in its non-monetary form, our model is not a dynamical selection procedure of the Walrasian correspondence.

The main result of this paper is the following: The state of an economy always converges towards some (locally) Pareto-optimal allocation provided the economy never falls into a liquidity trap. Speaking somewhat less loosely, Theorem 3 below exhibits an optimal growth rate of inside money $M_{t}$. If money grows sufficiently rapidly (given households' expectations and willingness to use their own private money), every trade path will converge to some (locally) Pareto efficient rest-point. Otherwise, sooner or later, the economy enters a liquidity trap, and stays there for good unless additional inside money is injected in the system. Unlike M. Friedman's golden rule, it turns out that the optimal growth rate of inside money depends upon the local gains-to-trade and households' expectations. Broadly speaking, suppose that at time $t$ households' expectations are too pessimistic. Then, there will be no-trade whatever being the local gains-to-trade available and the amount of circulating money: The economy sticks with its current state as long as households don't change their mind. Suppose, on the contrary, that all gains-to-trade have already been exhausted. Then, whatever being players' expectations and the quantity of circulating money, no-trade will again prevail. Finally, suppose that the amount of inside money, $M_{t}$, injected in the economy by the Bank is so tiny that the interest rate, $r(t)$, is sufficiently large to overthrow every attempt from the investors to take benefits from local gains to trade: Again, the economy remains stuck at some inefficient state due to the lack of liquidity.

\subsection{Nominal determinacy and liquidity}

We partly follow the monetary paradigm as set out by Dubey \& Geanakoplos (1992, 2003a,b) by considering households endowed with outside money together with a Central Bank injecting inside money. However, we depart from their framework in as much as 1) we allow agents to send limit-orders (and not just market orders) to the market, and

\footnotetext{
${ }^{8}$ Which solves Hahn's (1965) long-standing puzzle.

${ }^{9}$ In fact the whole dynamics is computable but we do not develop this feature here.

${ }^{10}$ See, e.g., Smale (1976).
} 
2) profits of the Central Bank from period $t$ are redistributed to private shareholders at time $t+d t$ (cf. Shubik \& Tsomocos (1992)). Hence, private shareholders cannot instantaneously use the dividends of the Bank from time $t$ as cash (outside money) in order to finance their purchases of time $t$.

In a related monetary framework, Drèze \& Polemarchakis $(1999,2000,2001)$ also assume that the Bank distributes its profits to private shareholders. Since they are in a static one-shot world however, shareholders can instantaneously use them to finance trades and pay their own debts to the Bank. As a consequence, there is no outside money in their model, and nominal indeterminacy of static equilibria is the rule. The exception is when the government budget constraint is violated, in which case Drèze \& Polemarchakis' model would also result in nominal determinacy. In a sense, violation of the government budget constraint is equivalent to existence of outside money. In the present paper, the governement "violates" its budget constraint during a nano-second, i.e., "between" $t$ and $t+d t$. Hence, in contrast with Dubey \& Geanakoplos (2003a,b), the exit of outside money is incipient in this paper because this money (being equal to the Bank's instantaneous profit) is reinjected in the economy every nano-second later. Thus, unlike Drèze \& Polemarchakis (2001, strong indeterminacy) and Dubey \& Geanakoplos (2003b, 2006, generic local uniqueness), we get (piecewise, generic) global uniqueness of the monetary trade paths both in real and nominal terms. ${ }^{11}$

Finally, our approach sheds some light on the crucial issue of quantitative measures of liquidity. Indeed, short-run interest rates turn out, in this paper, to play exactly the role of repo rates. We show that the speed of real trades is a function of the ratio of inside to outside money - itself equal to the repo rate. If this ratio is above a certain threshold, the speed of trades will be maximal - in fact, it will be equal to the speed of Walrasian trades in the barter version of our model (markets are perfectly liquid). If this ratio is too low, then the speed of trades decreases and current interest rate increases - so that markets become illiquid. Next, if the above ratio is below some critical value, then trades collapse. An important point is that the critical threshold just alluded to depends upon the size of local gains-to-trades and expectations. Put differently, the impact of the amount of circulating money on market liquidity cannot be evaluated independently of the real side of the economy: It is its interplay with (real) gains-to-trades and markets' expectations that makes markets more or less liquid.

The next section presents the dynamics in details. We begin with the stripped-down case of a barter exchange economy verifying standard interiority assumptions. Then, in section 3, money is introduced, and the interiority restriction is dropped. Section 4 is devoted to our main results. In order to focus on the essentials, and using the fact that markets are perfectly competitive, the game-theoretic foundation of the dynamics is not emphasized before section 5 where we flesh-out our model with the micro-structure underlying infinitesimal trades. The last section offers concluding remarks. The more technical proofs are relagated in the Appendix.

\footnotetext{
${ }^{11}$ For an excellent discussion of the issue of indeterminacy, see Bloise \& Polemarchakis (2006). See also Tsomocos (2008).
} 


\section{The dynamics}

The state of an economy is viewed as a pointlike object that moves along paths which are solution curves of a system of ordinary differential equations determined by various force fields. Three vector fields are interacting together: 1) a scalar measure, $\gamma(x)$, of the real gains-to-trade locally available at an arbitrary allocation of goods $x ;^{12}$ 2) a vector field characterizing households' expectations about the future; 3 ) the vector field of outside and inside money quantifying how money is indeed "the grease that turns the wheels of commerce".

\subsection{Barter economies}

Let us introduce each field separately. We first consider a barter economy $\mathcal{E}:=$ $\left(u_{i}, \omega_{i}\right)_{i=1, \ldots, N}$ verifying the following standard smoothness restrictions:

For each individual $i=1, \ldots, N, u_{i}: \mathbb{R}_{++}^{C} \rightarrow \mathbb{R}$ denotes a $\mathcal{C}^{2}$-utility, defined on his consumption set $\mathbb{R}_{++}^{C}$, while $\omega \in \mathbb{R}_{++}^{C}$ denotes his inital endowment stock. Suppose, for simplicity, that $u_{i}$ is smoothly strictly increasing, smoothly concave, and verifies the usual boundary assumption $\left\{x \in \mathbb{R}_{+}^{C}: u_{i}(x)=\right.$ $\left.u_{i}\left(\omega_{i}\right)\right\} \cap \partial \mathbb{R}_{+}^{C}=\emptyset$.

For this subsection, suppose, in addition, that, along a path of trades, investors have no expectation about the future, hence are entirely myopic (this in order to focus on the field of local gains-to-trade). When restricted to this class of economies, and under the myopia assumption, our dynamics becomes similar to the ones firtst analyzed by Champsaur \& Cornet (1990), Bottazzi (1994) and Giraud (2004) ${ }^{13}$. That is, the configuration set of the continous-time dynamics is given by the set of states $x(t):=$ $\left(x_{i}(t)\right)_{i} \in\left(\mathbb{R}_{++}^{C}\right)^{N}$; at each instant $t$, when the state is $x(t)$, individuals exchange infinitesimal trades in a tangent market $T_{x(t)} \mathcal{E}:\left\langle-x_{i}(t), \nabla u_{i}\left(x_{i}(t)\right)\right\rangle_{i}$. The latter is defined as a linear auxiliary economy with the same set of individuals $1, \ldots, N$, the same set of commodities $1, \ldots, C$, except that, now, each individual's set of trades is the shifted cone: $-x_{i}(t)+\mathbb{R}_{++}^{C}$, his initial endowment is 0 , and his short-run utility is:

$$
v_{i}\left(\dot{x}_{i}(t)\right):=\nabla u_{i}\left(x_{i}(t)\right) \cdot \dot{x}_{i}(t) .
$$

In other words, in the tangent market $T_{x(t)} \mathcal{E}$, trades are net, and $i$ 's short-sale upperbound is given by his current stock $x_{i}(t) .{ }^{14}$ Traders meet every time on the tangent market, myopically trading in the direction of the steepest increase of their own, current long-run utility. Each individual's budget set is

$$
p(t) \cdot \dot{x}_{i}(t) \leq 0, \text { and } \dot{x}_{i}(t) \geq-x_{i}(t),
$$

\footnotetext{
${ }^{12}$ This scalar was originally defined in Dubey \& Geanakoplos (2003a).

${ }^{13}$ See also Smale (1976b) for a seminal contribution along the same line.

${ }^{14}$ One can think of $x_{i}(t)$ as implicitly playing the role of $i$ 's collateral.
} 
while the macro-feasibility constaint on infinitesimal net trades is: $\sum_{i} \dot{x}_{i}(t)=0$. The cone of infinitesimal moves $\dot{x}_{i}(t)$ is then given by the set of Walrasian allocations of the linear economy $T_{x(t)} \mathcal{E}$, taking place at the corresponding competitive price. The dynamics is therefore given by the following ordinary differential inclusion:

$$
(\dot{x}(t), p(t)) \in \mathrm{WE}\left[T_{x(t)} \mathcal{E}\right], \quad x(0)=\omega, p(0) \text { arbitrary, }
$$

where $\mathrm{WE}\left[T_{x(t)} \mathcal{E}\right]$ is the subset of Walras equilibria of $\left[T_{x(t)} \mathcal{E}\right]$.

\section{Example 1.}

The following example illustrates this simple dynamics in the standard, myopic CobbDouglas case:

$$
T_{x(t)} \mathcal{E}:=\left\langle-x_{i}(t),\left(\sqrt{\frac{x_{i}^{2}(t)}{x_{i}^{1}(t)}}, \sqrt{\frac{x_{i}^{1}(t)}{x_{i}^{2}(t)}}\right)\right\rangle_{i}:
$$

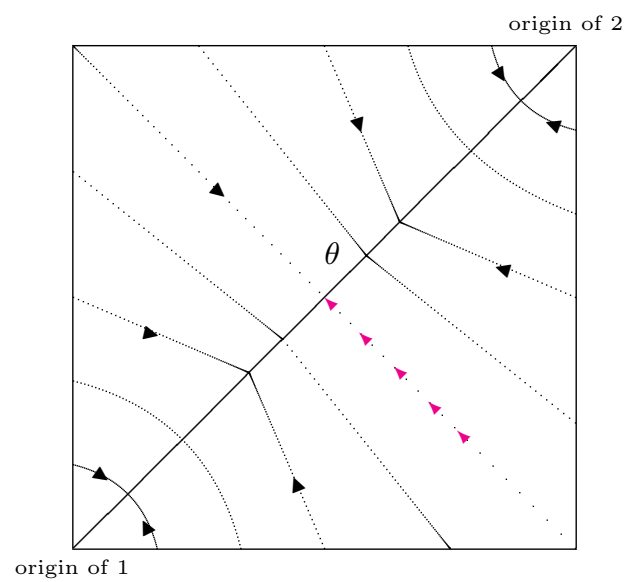

Fig. 1. The phase portrait of a barter, myopic Cobb-Douglas economy.

Here, the dynamics results in a smooth vector field, except on the diagonal of Pareto allocations $\theta$ which coincides with the set of singular points - where trades cease, whatever being the direction from which the economy touches $\theta$. It follows from the classical Cauchy-Lipschitz theorem that, given initial condition $\omega$, the dynamics admits a unique integral curve (trade path), which converges in finite time towards some efficient allocation in $\theta$ that depends smoothly upon $\omega$. Moreover, prices adjust smoothly along the trade path so as to be always orthogonal to the direction of move. Eventually, prices converge towards the unique decentralizing price vector that turns the limit-allocation into a price equilibrium.

At an arbitrary allocation of goods $x$, the crucial variable is the scalar measure $\gamma(x)$ of local gains-to-trade, which can be viewed, in the previous $2 \times 2$ example, as connected with the ratio, $(1+\gamma(x))^{2}$, of slopes of the (linearized) indifference curves at $x$ or, equivalently, with the ratio of agents' marginal rates of substitution for the two goods: 


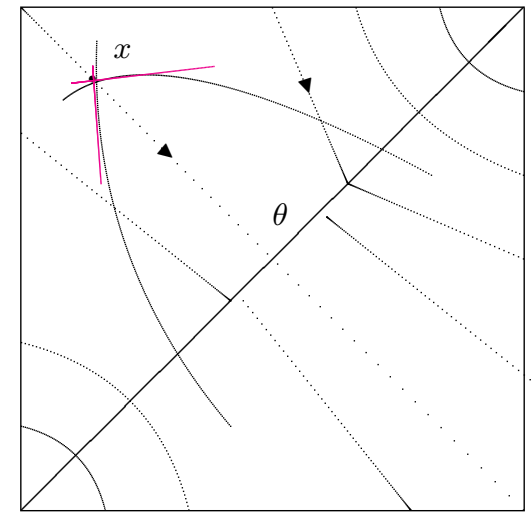

Fig. 2. $(1+\gamma(x))^{2}=$ the ratio of slopes of linearized indifference curves at $x$.

As long as $x$ does not reach $\theta, \gamma(x)$ can be seen as the force driving the state. On the contrary, when the contract curve $\theta$ is reached, $\gamma(x)=0$, and trades vanish. The next result is an immediate corollary of Theorem 3 below.

Proposition 1.-Under the standard smoothness restrictions on $\mathcal{E}$, (3) admits solution paths $(x(t), p(t))_{t}$, and each such trade path $(x(t))_{t}$ converges to some Pareto efficient point $x^{*} \in \theta$ while prices $(p(t))_{t}$ converge to some price vector $p^{*}$ sustaining $x^{*}$.

Notice that the whole dynamics is entirely ordinal in the sense that it is based solely on the local shape of households' indifference curves. It does not depend upon the utility functions $u_{i}$ used in order to represent households'preferences.

\subsection{Locally rational expectations}

Agents' $i$ expectations are captured through a saving function, $s_{i}: S_{+}^{C-1} \times \mathbb{R}_{++}^{C} \rightarrow \mathbb{R}_{+}^{C}$ which associates to current endowment, $x_{i}(t)$, a bundle of saved commodities: $0 \leq$ $s_{i}\left[x_{i}(t)\right] \leq x_{i}(t) .^{15}$

Let us denote by $\delta_{i}(t):=x_{i}(t)-s_{i}\left[x_{i}(t)\right]$ the bundle of commodities that, given his expectations, agent $i$ is ready to put on the market at time $t$. Of course, ${ }^{16}$

$$
0 \leq \delta_{i}(t) \leq x_{i}(t) .
$$

A tangent market $T_{x(t), \delta(t)} \mathcal{E}$ is defined in the same way as in the previous case, except that the budget constraint (2) is replaced by:

$$
p(t) \cdot \dot{x}_{i}(t) \leq 0, \text { and } \dot{x}_{i}(t) \geq-\delta_{i}(t),
$$

\footnotetext{
${ }^{15}$ For the sake of realism, we could let $s_{i}[\cdot]$ depend upon time and, say, upon some statistics over past prices. Besides leading to a non-autonomous dynamical system, this would not qualitatively change the results.

${ }^{16}$ The constraint (4) amounts to requiring investors to be able to physically exhibit their claimed collateral, which is consistant with the Shapley-Shubik approach (Giraud (2003)).
} 
When people's expectations become so pessimistic that $\delta_{i}(t)=0$, every $i$, then no-trade will occur in the tangent market. ${ }^{17}$ The myopic economy of the previous subsection corresponds to the particular no-saving case: $\delta_{i}(t)=x_{i}(t)$, every $t$ and $i$. The dynamics with expectations is now given by:

$$
(\dot{x}(t), p(t)) \in \mathrm{WE}\left[T_{x(t), \delta(x)} \mathcal{E}\right], \quad x(0)=\omega, \quad(p(0), \delta(0)) \text { arbitrary } .
$$

\section{Example 1 (continued)}

Back to the Cobb-Douglas workhorse, suppose that $\omega_{1}=(1,4), \omega_{2}=(4,1)$, and that, due to some shock in expectations, agent 1 anticipates, at $t$, an increase in the relative price of commodity $x^{2}$. He should increase the quantity of commodity $x^{2}$ saved for later use, so that $\delta_{1}^{x^{2}}(t)$ should decrease. The result, as can be readily checked, is that the relative price $p_{x^{2}}(t)$ will, indeed, be larger than it would have been, had player 1 not refrained from sending commodity $x^{2}$ to the market. As a consequence, 1's expectation has been momentarily confirmed, at least locally in time. This is the sense in which we shall say that investors, in this paper, entertain "locally rational expectations".

Next, if player 1 constantly anticipates $p_{x^{2}}(t)$ to relatively increase (in a broad sense) w.r.t. $p_{x^{1}}(t)$, while player 2 remains myopic, then, at time $t$, the tangent market will look like:

$$
T_{x(t), \delta(t)} \mathcal{E}:=\left\langle-\delta_{i}(t),\left(\sqrt{\frac{x_{i}^{2}(t)}{x_{i}^{1}(t)}}, \sqrt{\frac{x_{i}^{1}(t)}{x_{i}^{2}(t)}}\right)\right\rangle_{i},
$$

with $\delta_{2}(t)=x_{2}(t), \delta_{1}^{x^{1}}(t)=x_{1}^{1}(t)$, and $0<\delta_{1}^{x^{2}}(t)<x_{1}^{x^{2}}(t)$. As the following portrait phase shows, the resulting trade path (in red) will follow a curve quite different from its myopic benchmark (the green straight line), and end up in $\theta$ at a point that is more favorable to 1 .

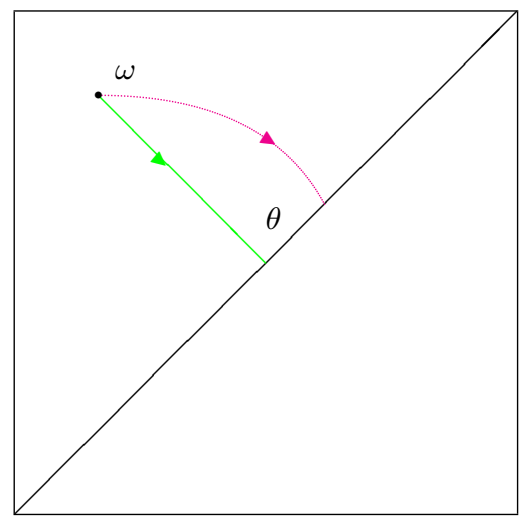

Fig. 3. The impact of expectations in the barter Cobb-Douglas economy.

\footnotetext{
${ }^{17}$ This, in a way, echoes the well-known "autarkic equilibrium" of strategic market games, cf. Giraud (2003). It is not unrelated to the "trauma" currently faced by markets for exotic assets, and especially for credit derivatives.
} 
Notice that, along the trade path, the relative price of commodity $x^{2}$ constantly decreases with respect to the price of $x^{1}$. Hence, although they are locally confirmed (in the sense that $p_{x^{2}}(t)$ would decrease more rapidly if player 1 was not anticipating it to increase), 1 's expectations are globally contra-cyclical in this example. Notice also that, if, on the contrary, 1 entertains pro-cyclical expectations about the price of commodity $x^{2}$, the resulting trade-path will be identical to the myopic benchmark (this property, of course, is peculiar to this example). Finally, when the economy touches $\theta$, trades vanish and prices remain constant, so that the investors' expectations may still be regarded as being locally confirmed. Hence, there is a genuine interplay between the field of local gains-totrade and that of expectations. To put it somewhat vaguely: the larger the local gainsto-trade, the stronger the impact of expectations. Direct verification also shows that, whenever the two agents of the Cobb-Douglas economy have "isomorphic" expectations (in the sense that there exists a common $0<\lambda \leq 1$ such that $\delta_{i}(t)=\lambda x_{i}(t), i=1,2$, every $t$ ), then the resulting trade paths are identical to those of the myopic case, except that the speed of trades is reduced by a factor $\lambda$. Indeed, isomorphic savings on the part of both traders amounts to rescaling the unit of commodities in every tangent market, say from pounds to ounces, but leaves invariant the direction of moves. This captures a phenomenon observed again during the recent subprime crisis: the lack of confidence in the future may induce a decrease in the velocity of trades. Moreover, it implies that the "myopic" trade path can be adopted as a benchmark, with respect to which differences in actual trade paths are only due to differences in traders' expectations. The next result is also a straightforward corollary of Theorem 3 below.

Proposition 2.- Under the standard smoothness restrictions, (6) admits solution paths. Provided that, for every household $i$, expectations verify: $\delta_{i}(t)>0$ a.e. $t$, every such trade path $(x(t))_{t}$ converges to some Pareto efficient point $x^{*} \in \theta$ while prices $(p(t))_{t}$ converge to some corresponding sustaining price $p^{*}$.

\section{Monetary economies}

We now introduce money and drop the standard smoothness restrictions. Throughout the rest of this paper, the real long-run economy is defined by $\mathcal{E}=\left(u_{i}, \omega_{i}\right)_{i}$, with $\omega_{i}>0$, $\bar{\omega}:=\sum_{i} \omega_{i} \gg 0$, each $u_{i}$ being differentiable over the subset $X_{i}=\left\{x_{i} \in \mathbb{R}_{+}^{C} \mid \exists x_{-i} \in\right.$ $\left.\left(\mathbb{R}_{+}^{C}\right)^{N}:\left(x_{i}, x_{-i}\right) \in \tau\right\}$ with

$$
\tau:=\left\{x \in\left(\mathbb{R}_{+}^{C}\right)^{N} \mid \sum_{i} x_{i}=\bar{\omega} \text { and } u_{i}\left(x_{i}\right) \geq u_{i}\left(\omega_{i}\right) \forall i\right\}
$$

being the subset of feasible and individually rational allocations. Throughout the rest of this paper, we also suppose that, whenever defined, each gradient verifies: $\nabla u_{i}\left(x_{i}\right)>0$.

Regarding the monetary sector of the economy, at time $t$, each agent $i$ has some private endowment $m_{i}(t)$ of outside fiat money, of which he is ready to put $\mu_{i}(t) \leq$ 
$m_{i}(t)$ on the tangent market. Given his expectations, he may indeed choose to keep some precautionary quantity of outside money in his pocket - in which case, $\mu_{i}(t)<$ $m_{i}(t)$. Since default is not allowed in this paper, $\mu_{i}(t) \geq 0$ anyway, every $i$ and every $t$. Households' expectations are thus defined through a saving function, $s_{i}: \mathbb{R}_{+}^{C} \rightarrow \mathbb{R}_{+}^{C+1}$ which associates to each current endowment, $x_{i}(t)$, a bundle of saved commodities: $s_{i} \leq x_{i}(t)$, and a scalar cash-holding $\sigma_{i}(t):=m_{i}(t)-\mu_{i}(t)$.

At each time $t$, the quantity $M(t) \geq 0$ of inside money is injected in the system by the Central Bank, according to some exogenously given monetary policy. Outside money is owned by households free and clear of debt. Inside money is always accompanied by debt when it comes into households' hands. The aggregate stock of outside money held by investors at time $t$ is $\bar{m}(t)=\sum_{i} m_{i}(t)$. The aggregate flow of money that investors are ready to put on the market at time $t$ is $\bar{\mu}(t)=\sum_{i} \mu_{i}(t)$. Let $r(t) \in \mathbb{R}_{+}$stand for time's $t$ intra-period (or instantaneous) rate of interest on the Bank loan.

In the rest of this subsection, we provide some details about monetary infinitesimal trades. The impatient reader may immediately move on to subsection 2.3. now, where a reduced-form of our dynamics is to be found.

\subsection{The budget set with fiat money}

Infinitesimal trades take place on the monetary tangent market $T_{z(t)} \mathcal{E}$, defined as a monetary linear economy with $N$ agents and $C$ commodities, each agent being equipped with the cone, $-\delta_{i}(t)+\mathbb{R}_{+}^{C}$, of infinitesimal trades, 0-endowment in commodities, $m_{i}$ Euros in his pocket, and the short-run utility, $v_{i}(\cdot)$, defined by (1).

For the sake of clarity, consider each instant $t$ as being divided into three subperiods. In the first one, say $t_{\alpha}$, investors borrow inside fiat money from the Bank, say by selling IOU notes or bonds. The quantity of inside money borrowed by agent $i$ depends upon his expectations, the quantity $\mu_{i}(t)$ of outside money he decided to spend and on the stock, $M(t)$, of inside money injected by the Bank. In the second subperiod, $t_{\beta}$, households trade real commodities against money. In the third, $t_{\gamma}$, they repay Bank loans with money according to the rate $r(t)$. All commodity markets meet simultaneously in subperiod $t_{\beta}$. The difference with the barter case is that, now, households are imposed a cash-in-advance constraint, that is, they have to pay money in order to purchase commodities. It is only in the third interval, after commodity markets close, that revenue from the sales of commodities come into households' hands, by which time it is too late to use these revenues for purchase. Those households who, at time $t_{\alpha}$, find their cash available for trades, $\mu_{i}(t)$, insufficient (say, because of their expectations about future prices or about future interest rates) will therefore need to borrow money from the Bank in order to finance purchases, and will defray the loan out of their sales revenues.

The price of money at time $t_{\alpha}$ is $\frac{1}{p_{c}(t)}$ in terms of commodity $c$, and $(1+r(t))$ in terms of money at time $t_{\gamma}$. As $r(t) \rightarrow-1$, money-now (i.e., at time $t_{\alpha}$ ) loses all value in terms of money-later (at time $t_{\gamma}$ ). Conversely, when $r(t) \rightarrow+\infty$ money-later loses all value in terms of money-now. Finally, when $r(t)=0$, money-now and money-later become equal. 
Suppose that agent $i$ borrows $\tilde{m}_{i}(t)$ Euros at time $t_{\alpha}$ by promising to pay $(1+$ $r(t)) \tilde{m}_{i}(t)$ at $t_{\gamma}$, after commodity trades. Since trades are net, the budget constraint now is (compare with (5)):

$$
p(t) \cdot \dot{x}_{i}(t) \leq \tilde{m}_{i}(t)+\mu_{i}(t) \text { and } \quad \dot{x}_{i}(t) \geq-\delta_{i}(t) .
$$

Next, at time $t_{\beta}$, all commodities are traded simultaneously, and the cash-in-advance constraint of agent $i$ is given by: ${ }^{18}$

$$
p(t) \cdot \dot{x}_{i}^{+}(t) \leq \tilde{m}_{i}(t)+\mu_{i}(t)
$$

which says that total money spent on purchases cannot exceed the money on hand, i.e., money borrowed plus money endowed (and not deliberately saved as cash-holding). Notice that (9) implies the first part of (8). We therefore synthetize both constraints into a single "monetary constraint":

$$
p(t) \cdot \dot{x}_{i}^{+}(t) \leq \tilde{m}_{i}(t)+\mu_{i}(t) \text { and } \quad \dot{x}_{i}(t) \geq-\delta_{i}(t)
$$

Outside trade curves that are solutions of our dynamics, it may well be the case that some investor happens to be surprised by unforseen prices $p(t)$ or by an unexpected interest rate $r(t)$, and hence need to draw money from his saving $\sigma_{i}(t)$ in order to be able to deliver on his loan. In case his saving was zero $\left(\mu_{i}(t)=m_{i}(t)\right)$, then this household would be forced to default. According to the definition of locally rational expectations given in this paper, this never happens along a trade path solving our dynamics: Every household must fully deliver on its loan at every time without touching his hoarded cash-holding at this very period, i.e., ${ }^{19}$

$$
(1+r(t)) \tilde{m}_{i}(t) \leq p(t) \cdot\left[\dot{x}_{i}(t)\right]^{-}+\Delta(9),
$$

where $\Delta(9)$ is the difference between the right- and the left-hand sides of inequality (9). Given $\left(\delta^{i}(t), \mu^{i}(t)\right) \in \mathbb{R}_{+}^{C+1}$ and market prices $(p(t), r(t)) \in \mathbb{R}_{+}^{C+1}$, the budget set $B\left(p(t), r(t), \delta^{i}(t), \mu^{i}(t)\right)$ of household $i$ on monetary tangent markets at time $t$ consists of all market actions $\left(\tilde{m}_{i}(t), \dot{x}^{i}(t)\right)$ that satisfy (10) and (11).

At the macro-level, monetary infinitesimal trades in the monetary tangent market are feasible when they verify the following clearing equations:

$$
\sum_{i} \tilde{m}_{i}(t)=M(t)
$$

and

$$
\sum_{i} \dot{x}_{i}(t)=0
$$

\footnotetext{
${ }^{18}$ Here, $x^{+}:=\max \{x, 0\}$.

${ }^{19} x^{-}:=\max \{-x, 0\}$.
} 


\subsection{Interest rate: basic properties}

The commodity market clearing condition (13) guarantees that the total stock of commodities $x(t)=\left(x_{i}(t)\right)_{i}$ is conserved and redistributed among consumers according to current infinitesimal trades - that is, the economy never leaves the boundary of the feasible set $\tau$ (see (7)). And (13) multiplided by $p(t)$ shows that the aggregate flow of money used for trades, $\sum_{i}\left(\tilde{m}_{i}(t)+\mu_{i}(t)\right)$, is conserved and also redistributed among investors as counterpart of their real trades. Thus, after trades have occured within time $t$, all of $M(t)+\bar{m}(t)$ is with households. The no-default condition (11) then implies that the total debt of households does not exceed $M(t)+\bar{\mu}(t)$. At the end of time $t$, the Bank holds $(1+r(t)) M(t) \leq M(t)+\bar{\mu}(t)$, and investors hold the balance plus their own, private precautionary money saving: $\bar{\mu}(t)-r(t) M(t)+\sum_{i} \sigma_{i}(t)$. Hence, the instantaneous profit of the Bank at time $t$ is $r(t) M(t)$. On the other hand, the aggregate stock of outside cash available for trades, $\bar{\mu}(t)$, remains constant across the various time intervals within $t$, whoever it belongs to. Hence, at least $M(t)+\bar{\mu}(t)$ is owed to the Bank. Along a trade path solving our dynamics, no more can be owed to the Bank since investors never need to draw money from their saving (cf. (11)). Whence $(1+r(t)) M(t)=M(t)+\bar{\mu}(t)$, i.e. ${ }^{20}$

$$
r(t)=\frac{\bar{\mu}(t)}{M(t)} .
$$

This shows that the interest rate $r$ in our monetary dynamics is determined by the interaction between the real and the monetary sector solely through the interplay between the stocks of inside and outside money together with households' expectations.

Obviously, we must have $0 \leq \tilde{m}_{i}(t) \leq M$. We shall impose as part of the trading rules on each tangent market that, whenever $\bar{\mu}(t) \neq 0$ :

$$
\tilde{m}_{i}(t):=\frac{\mu_{i}(t)}{\bar{\mu}(t)} M(t)=\frac{\mu_{i}(t)}{r(t)} .
$$

This can be justified as a weakening of the rational expectations hypothesis, applied to period $t$. Suppose, indeed, that investors were able to perfectly anticipate $p(t)$ and $r(t)$ from period $t_{\alpha}$ on. On account of their being no inter-period charged interest rate, after repaying the Bank at $t_{\gamma}$, no household would be left with more money, $\sigma_{i}(t)$, than it decided to save (given his rational expectations), otherwise it should have spent more money at time $t_{\beta}$ to purchase commodities, or else curtailed its sales, improving its short-run welfare. Hence, the no-default constraint (11) should be satisfied as an equality:

$$
p(t) \cdot \dot{x}_{i}(t)=\mu_{i}(t)-r(t) \tilde{m}_{i}(t) .
$$

A little reflections shows that the right-hand side of (16) would also be nonnegative. Indeed, suppose the contrary: This means that player $i$ would have to finance part of the cost, $r(t) \tilde{m}_{i}(t)$, of his loan by his sales $p \cdot \dot{x}_{i}(t)$. But he could do so, again, by

\footnotetext{
${ }^{20}$ With the convention $\frac{0}{0}:=0$.
} 
borrowing less money (i.e., selling a smaller quantity of bonds to the Bank for inside money), and saving a larger part of his current endowment, improving again his shortrun welfare. Now, (16), (13) and $\mu_{i}(t)-r(t) \tilde{m}_{i}(t) \geq 0$, every $i$, imply (15) for every household $i$. In this paper, we shall not assume that investors share perfect foresight within each period $t$, but we simply impose (15). ${ }^{21}$

As a consequence of (15), the no-default condition (11) can now be written as a standard budget constraint in net trades:

$$
p(t) \cdot \dot{x}_{i}(t) \leq 0
$$

so that the various monetary constraints faced by agent $i$ can be summarized by (10) and (17). Obviously, when $\mu_{i}(t)=0, i$ must finance his trades with borrowed money. If, however, $\bar{\mu}>0$, then $\tilde{m}_{i}=0$, and player $i$ cannot trade. If, on the other hand, $M(t)=0$, there is no Bank money available, and no-trade prevails for everybody. Next, when $M(t) \rightarrow+\infty$, the interest rate vanishes, so that (11) is trivially satisfied, as well as (9) (up to a normalization of prices), and (8) becomes the unique remaining constraint. We are then back to the barter case. Finally, if investor $i$ anticipates an increase in the interest rate, he should save less money at time $t_{\alpha}$, since, ceteris paribus, he will have to pay more for his loans. And, indeed, an increase in $\mu_{i}(t)$ induces an increase in $r(t)$ as shown by (14) - another local confirmation of the rationality of $i$ 's expectations.

What happens to the Bank's current profit, $r(t) M(t)=\bar{\mu}(t)$ at time $t$ ? We assume, in this paper, that it is distributed at $(t+d t)_{\alpha}$ to its private shareholders according to some fixed ownership structure $\left(\nu_{i}\right)_{i} \in(0,1]^{N}$, such that $\sum_{i} \nu_{i}=1$. Therefore, the whole stock of outside money $\bar{\mu}(t)$ that was initially put on the market by households will return to them in the form of dividends. As a consequence, variations in the aggregate amount, $\bar{\mu}(t)$, of outside money available for trades are solely due to the investors' expectations: Whenever those expectations are constant across time, the stock $\bar{\mu}(t)$ remains constant. Moreover, the rate $r(t)$ is determined solely by the aggregate amounts, $\bar{\mu}(t)$ and $M(t)$, of outside and inside money available for trades. It is therefore affected by the real sector only through the canal of expectations. ${ }^{22}$ On the other hand, the cash received from the Bank as dividends at time $t$ (arising from profits induced by time $t_{\alpha}$ infinitesimal loans) is received by shareholders only at time $(t+d t)_{\alpha} \cdot{ }^{23}$ Consequently, the stock of outside money hold by agent $i$ must satisfy the following differential equation:

$$
\dot{m}_{i}(t)=\nu_{i} r(t) M(t)-\mu_{i}(t)-p(t) \cdot \dot{x}_{i}(t)=\nu_{i} \bar{\mu}(t)-\mu_{i}(t)-p(t) \cdot \dot{x}_{i}(t) .
$$

Indeed, according to (16), $p(t) \cdot \dot{x}_{i}(t)$ is the amount of cash that may have been lost by agent $i$ at the end of period $t$. It will turn out, however, that along a trade curve solving

\footnotetext{
${ }^{21}$ It can also be interpreted as follows: at $t_{\alpha}$, each investor $i$ sends $\mu_{i}$ Euros to the clearing house as a collateral in order to borrow money from the Bank, and is served at the prorata of his contribution to the $\operatorname{sum} \bar{\mu}$ of outside cash.

${ }^{22} \mathrm{~A}$ noteworthy consequence is that changes in the interest rates are solely due to changes in expectations. Hence, investors' expectations about $r(t)$ can be viewed in our model as expectations about their opponents' changes in expectations.

${ }^{23}$ A general (static) formulation where shares of ownership are endogenously determined can be found in Shubik \& Tsomocos (1992).
} 
our dynamics, (16) is verified as an equality, so that the dynamics of individual endowed money reduces to:

$$
\dot{m}_{i}(t)=\nu_{i} \bar{\mu}(t)-\mu_{i}(t) .
$$

\subsection{A local Quantity Theory of Money}

Summing (10) over $i$, and using (12) yields the following (localized) version of Irwing Fisher's celebrated quantity theory of money:

$$
\bar{\mu}(t)+M(t) \geq p(t) \cdot\left(\sum_{i} \dot{x}_{i}^{+}(t)\right)
$$

provided trades are effective, that is: $\sum_{i} \dot{x}_{i}^{+}(t) \neq 0 .{ }^{24}$ Whenever households perfectly anticipate $(p(t), r(t))$ so that (10) is binding for every $i$, then (20) will be satisfied as an equality. This, however, need not always be the case along a trade curve solving our dynamics, as the local rationality of households' expectations is too weak to ensure that no household will never use less money to finance its purchases than it initially intended to. Thus, people's bounded rationality is responsible for (20) being only a weak inequality in general. We shall see, nevertheless, that this does not prevent our monetary theory from exhibiting "monetarist" features under certain circumstances that are specified by Theorem 3 below.

Notice that, in (20), income corresponds to infinitesimal sales and not to initial endowments. Moreover, at variance with the textbook analysis of Fisher's equation, and apart from the quantity $M(\cdot)$ of inside money, (20) only involves endogeneous variables: infinitesimal trades $\dot{x}(t)$, as well as prices $p(t)$ and available cash $\bar{\mu}(\cdot)$ are all determined by the three forces at work - local gains-to-trade, expectations and money. Being stated in the tangent bundle of $\tau$, our quantity theory of money involves only flows (and no stock). Finally, the velocity, $V$, of money is often understood as being given by

$$
M V=P T .
$$

When compared literally, (20) and (21) might seem to mean that $V$ is constantly equal to 1 in our theory. This is not the interpretation followed here. Rather, we consider that the velocity of circulating money is not constant in general, and can be measured by the speed of trades, $\left\|\sum_{i} \dot{x}_{i}^{+}(t)\right\|$, which is in turn endogenously determined by the interaction of gains-to-trade, expectations and money. More precisely, the money velocity depends upon the quantity of money spent: $\bar{\mu}(t)+M(t) \cdot{ }^{25}$ As more money is spent, the volume of infinitesimal trades increases, and so does the velocity of money. Conversely, when the amount of money saved (for precautionary or speculative purposes, given investors'

\footnotetext{
${ }^{24}$ When this latter condition is not satisfied, no-trade prevails, prices are indeterminate and the QTM breaks down.

${ }^{25}$ A similar interpretation is suggested by Dubey \& Geanakoplos (2003a). Equivalently, $\bar{\mu}(t)$ can be interpreted as depending upon fiscal policy and $M(t)$ upon monetary policy, so that these two classical tools can be related to the velocity of money.
} 
expectations) increases, the speed of trades decreases, and so does the velocity of money: It can even reduce to 0 , in which case the economy enters a liquidity trap. We will show (see Theorem 3 below) that, under certain circumstances, a change in $M$ (money stock) might be absorbed by an offsetting change in $V$ (velocity of circulation) and therefore may not be transmitted to $P$ (price level). Likewise, a change in income or the volume of market transaction might be accommodated by a change in velocity without requiring any change in the money supply. Restated in the setting of (20), it turns out that, at any feasible state, there is a threshold at which an increase of $M(t)$ (or $\bar{\mu}(t)$ ) results in an increase of the speed of trades and not in an increase of prices. Then, however, any further increase of money will induce inflation and no real effect. Thus, (20) turns out to induce a doctrinal mixture where both Keynesian and monetarist viewpoints are "locally" right.

\subsection{Monetary flows}

At each time $t$, the state of the monetary economy $\mathcal{E}$ is the collection

$$
z(t):=(x(t), \delta(t), m(t), \mu(t), M(t)) .
$$

The configuration space, $\mathcal{M}:=\tau \times\left(\mathbb{R}_{+}^{C+1}\right)^{N} \times \mathbb{R}_{+}^{N+1}$, is the set of feasible states of our dynamics, i.e., of feasible allocations in commodities, savings, and stocks of money $(x, \delta, m, \mu, M)$ with $\sum_{i} m_{i}=\bar{m}, 0 \leq \delta_{i} \leq x_{i}$ and $0 \leq \mu_{i} \leq m_{i}$.

\subsubsection{The need for a new solution concept}

Despite its long-standing tradition, the standard Walrasian allocation can no longer serve as a solution concept for tangent markets. Indeed, it is well-known that, whenever agents do not have strictly increasing preferences and interior endowments, Walrasian allocations may fail to exist in linear economies. The restriction $\delta_{i}(t)>0$ (a.e. $t$ ) used in Proposition 2 to avoid this situation is obviously too strong, as it imposes some form of long-standing "optimism" on the part of every agent that conflicts with everyday experience, at least since 2008. On the other hand, even whenever they exist, Walras equilibria are usually not unique (even though the corresponding normalized competitive price is unique ${ }^{26}$ ). So that there is no hope for recovering a unique solution path out of the differential inclusion (6). Thus, we shall replace the simple Walrasian concept by a generalized equilibrium solution, introduced by Mertens (2003), and tailored-made for linear economies. This will provide us with a more accurate and operational definition of infinitesimal flows $\dot{x}$ and prices $p$ for every tangent market $T_{x}(\mathcal{E})$.

Mertens' (2003) solution concept can be alternatively viewed as a stylized double auction $^{27}$ or as a member of the family of hierarchic equilibria ${ }^{28}$. Since its definition may look rather "dry", and will be further complicated by the introduction of the monetary

\footnotetext{
${ }^{26}$ For all these classical results regarding linear economies, cf. Cornet (1989).

${ }^{27}$ Its game-theoretic interpretation is provided in section 5 below.

${ }^{28}$ see Florig (2001).
} 
field, let us begin by presenting it by a couple of informal remarks. First, it is restricted to linear exchange economies. This, however, is harmless since, in this paper, tangent markets are already linear. Second, every traditional competitive equilibrium of a linear economy is still a solution in the sense of Mertens' (2003). Third, the latter yields a non-vacuous solution concept even when Walras allocation fail to exist.

\section{Example 2.}

Consider the following two-agent tangent market with

$C=2, v_{1}\left(\dot{x}_{1}, \dot{x}_{2}\right)=\dot{x}_{1}, v_{2}\left(\dot{x}_{1}, \dot{x}_{2}\right)=\dot{x}_{2}, \delta_{1}=(1,0), \delta_{2}=(2,3)$.

This economy admits no Walras allocation, and a little reflection reveals that the unique "reasonable" outcome should be no-trade. Indeed, agent 2 is solely interested in commodity $x_{2}$, while agent 1 decided to save his entire endowment in commodity $x_{2}$, hence has a zero short-sale upper-bound in this very commodity. This means that agent 1 refuses to sell any positive amount of the unique commodity that his counterpart is ready to buy. The situation is plotted in the Edgeworth box of Figure 2 (where $g_{i}$ is the gradient vector of agent $i$ ):

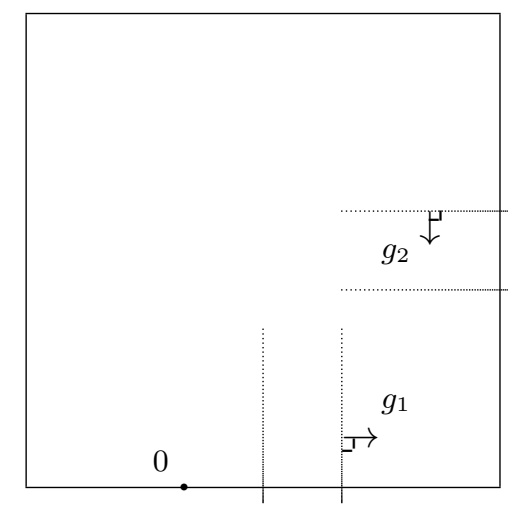

Fig. 4. A tangent market with no Walras allocation.

Mertens' (2003) solution for this example coincides with no trade, while competitive equilibria simply fail to exist.

\subsubsection{Pseudo-flows}

Let's now formally define the flow induced by the local interaction of traders within the double auction regulating trades in the monetary tangent market $T_{z(t)} \mathcal{E}$. It describes the flow of commodities at time $t$-hence the move, $\dot{x}(t)$, that characterizes changes in the households' stocks of commodities - as well as the instantaneous price vector at which these infinitesimal changes occur. ${ }^{29}$ To simplify notations, we drop the time index in this subsection. On the other hand, the finite set of traders is interpreted as a finite set of types, each type being represented by a unit interval, [0,1], of identical individuals,

\footnotetext{
${ }^{29}$ The flow of outside money is given by (18). That of inside money, $\dot{M}(t)$, is exogenously defined by the Central Bank's policy, as we differ the strategic analysis of the Bank's behavior to a subsequent work.
} 
equipped with the restriction, $\lambda$, of the Lebesgue measure. The set of individuals is therefore $\left([0,1]^{N}, \lambda^{\otimes N}\right)$.

Definition 1. (a) A monetary pseudo-flow of $T_{z} \mathcal{E}$ is a price, $p \in \mathbb{R}_{+}^{C} \backslash\{0\}$, a feasible $N$-tuple of borrowed money $\left(\tilde{m}_{i}\right)_{i} \in \mathbb{R}_{+}^{N}$, and a feasible infinitesimal net trade in commodities, $\dot{x} \in\left(\mathbb{R}_{+}^{C}\right)^{N}$, such that:

(i) For every individual $i, p \cdot \nabla u_{i}\left(x_{i}\right)=0$ implies $\dot{x}_{i}=0$. Moreover, if $\bar{\mu}>0$, then $\tilde{m}_{i}$ verifies $(15)$ for every $i: \tilde{m}_{i}=\left(\mu_{i} / \bar{\mu}\right) M$.

(ii) For every $i, \dot{x}_{i}$ maximizes $v_{i}(\dot{x})$ subject to the cash-in-advance (10) and budget constraints (17):

$$
\dot{x} \geq-\delta_{i}, \quad p \cdot \dot{x} \leq 0, \quad p \cdot \dot{x}^{+} \leq \tilde{m}_{i}+\mu_{i}, \text { and }\left(p^{c}=0 \Rightarrow \dot{x}_{i}^{c}=0\right) .
$$

(iii) For every commodity $c, p^{c}=0$ implies that, for a.e. $i,\left(p \cdot \delta^{i}>0 \Rightarrow\right.$ $\left.v_{i}^{c}=0\right)$.

(b) $(p, \dot{x})$ is a first-order pseudo-flow if furthermore a.e. trader $i$ maximizes his utility $v_{i}$ subject to (22), i.e., if $p^{c}=0 \Rightarrow v_{i}^{c}=0$ for a.e. $i$.

If $\mu_{i}=0$ while $\bar{\mu}>0$, then $i$ has no endowed money and can no more borrow Bank money (because of (15)). Hence, the cash-in-advance constraint (10) implies that $i$ is excluded from trades. On the other hand, when restricted to the subset of actors $\left\{i: p \cdot \delta_{i}>0\right.$ or $\left.\left(p_{c}=0 \Rightarrow v_{i}^{c}=0\right)\right\}$ (in particular, when $p \gg 0$ or $\nabla u_{i}\left(x_{i}\right) \gg 0$, every $i)$, then every pseudo-flow $(p, \dot{x})$ is of first-order. If, in addition, $\bar{\mu}=0$ but $M>0$, then $r=0$, the cash-in-advance constraint vanishes and $(p, \dot{x})$ reduces to a pair of Walras allocations (in net trades) and price ratios of the linear economy $T_{z} \mathcal{E}$. The same outcome obtains dually whenever $M \rightarrow \infty$ while $\bar{\mu}>0$. Then, indeed, $r \rightarrow 0^{+}$and, at the limit, the final infinitesimal trades induced by a pseudo-flow are not different from the Walrasian net trades obtained in $T_{z} \mathcal{E}$ in an idealized world without money at all, where prices only have the meaning of exchange rates between pairs of commodities. Finally, if $\bar{\mu}=M=0$, no-trade is the unique outcome.

The first virtue of monetary pseudo-flows, however, is that they exist under much more general circumstances than competitive equilibria, as shown by Lemma $1 .{ }^{30}$

\footnotetext{
${ }^{30}$ Pseudo-flows are also close to the concept of "monetary equilibrium" defined by Dubey \& Geanakoplos (2003a). The differences are the following: (a) Pseudo-flows are tailored only for linear economies while monetary equilibria make sens for more general concave economies. (b) Equation (15) defining borrowed money is imposed for $\bar{\mu}>0$ at a pseudo-flow. (c) The basic Shapley \& Shubik (1977) mechanism which serves to define prices and allocations (given offers and bids) at a monetary equilibrium is replaced, here, by Mertens' (2003) limit-price mechanism. These changes provide us with a solution even in situations where monetary equilibria would fail to exist and which is globally unique in most cases of interest.
} 
Lemma 1.- (a) Every monetary tangent market $T_{z} \mathcal{E}$ admits a monetary pseudo-flow.

(b) Every such pseudo-flow verifies: $p \cdot \dot{x}_{i}=0, \quad$ a.e. $i$.

Proof of Lemma 1. (a) If $\bar{\mu}=0$ and $M=0$, no-trade is the unique pseudo-flow. If $\bar{\mu}=0$ and $M>0$, a monetary pseudo-flow boils down to a "pseudo-equilibrium" (in the sense of Mertens (2003)) of $T_{z} \mathcal{E}$, expressed in net trades. Existence of such pseudo-equilibria follows from Mertens (2003, Lemma 3). If, now, $\bar{\mu}>0$, then all the traders $i$ for whom $\mu_{i}=0$ can be ignored since $\dot{x}_{i}=0$. Consider the restriction of the linear economy $T_{z} \mathcal{E}$ to those individuals $i$ with $\bar{\mu}_{i}>0$. If, in Definition 1(i), the cash-in-advance constraint $p \cdot \dot{x}^{+} \leq \tilde{m}_{i}+\mu_{i}$ is temporarily omitted, then the part $(p, \dot{x})$ of a monetary pseudo-flow reduces, once again, to a "pseudo-equilibrium" in net trades. Such a pseudo-equilibrium is defined up to a normalization constant $\lambda>0$ for prices. It therefore only remains to check that we can choose $\lambda$ so that $\lambda p \cdot \dot{x}^{+} \leq \tilde{m}_{i}+\mu_{i}$ is fulfilled for every $i$. This is easy since, by construction, $\tilde{m}_{i}+\mu_{i}>0$ for every $i$ (in the restricted economy).

(b) According to Def. 1(ii), $p^{c}=0 \Rightarrow \dot{x}_{i}^{c}=0$. Thus, we can ignore commodities with zero price, i.e., we assume $p \gg 0$. It then follows from the short-run utility maximization of Def1.(ii) that $p \cdot \dot{x}_{i}=0$.

The equality $p \cdot \dot{x}_{i}=0$ means that, along a trade curve solving our dynamics, an investor $i$ is never forced to spend more money $\mu_{i}(t)$ than he initially decided to (for a.e. time $t$ ). This confirms our rewriting of the dynamics of individual outside money (19), hence the fact that $\bar{m}(t)$ remains constant across time.

\subsubsection{Proportional rationing}

The price to pay for the previous easy existence proof is the huge indeterminacy of pseudo-flows. We shall therefore impose two restrictions: Taken together, they will provide us with the desired global uniqueness of the flow for each tangent market. What Definition 1 actually captures is how the price is determined by the clearing house ("teneur de marché") computing the market supply and demand functions, and settling at the intersection. In case of several possible quantities on the intersection, our first restriction is to assume that the clearing house follows a "proportional rationing": ${ }^{31} \mathrm{All}$ buyers whose (short-run) utility equals the market price get their orders executed in the same proportion, and similarly for all sellers. Formally, a monetary pseudo-flow is proportional whenever it verifies:

For every pair of items $\left(c, c^{\prime}\right) \in \mathbb{N}_{C+1}$, with non-zero prices, there exists $m_{c c^{\prime}} \geq 0$ s.t.

a) $m_{c c^{\prime}}+m_{c^{\prime} c}>0$

b) $m_{c_{1} c_{2}} m_{c_{2} c_{3}} m_{c_{3} c_{1}}=m_{c_{1} c_{3}} m_{c_{3} c_{2}} m_{c_{2} c_{1}}$ (consistency);

\footnotetext{
${ }^{31}$ See Mertens (2003) for a game-theoretic foundation of the proportionality rule.
} 
c) all agents $i$ with non-zero utility whose demand set verifies $D_{p}^{i}\left(\nabla u_{i}\right) \ni$ $\left\{c, c^{\prime}\right\}$ receive commodities $c$ and $c^{\prime}$ in quantities proportional to $m_{c c^{\prime}}$ and $m_{c^{\prime} c}$, where the demand set of $i$ at price $p$ is

$$
D_{p}^{i}\left(\nabla u_{i}\right):=\left\{\ell \mid p_{\ell} \leq\left(\frac{\partial u_{i}}{\partial x_{\ell}} / \frac{\partial u_{i}}{\partial x_{k}}\right) p_{k}, \quad \forall k=1, \ldots, C\right\} \cdot{ }^{32}
$$

In Example 2 supra, no-trade is also a proportional pseudo-flow: condition c) above is vacuously satisfied. In the next example, the proportional rule is effectively at work:

Example 3. $g_{1}=g_{2}=(1,1), \delta_{1}=(2,1), \delta_{2}=(1,3)$. Suppose, for simplicity, that $r=0$. Then, $P\left(T_{z} \mathcal{E}\right)=\{(1,1)\}$ while the unique proportional first-order pseudo-flow lies at the intersection of the Pareto set with the first diagonal of the Edgeworth box. More precisely, the weights are $m_{x y}=3$ and $m_{y x}=4$, and $\dot{x}_{1}^{*}=\left(\frac{9}{7}, \frac{12}{7}\right), \dot{x}_{2}^{*}=\left(\frac{12}{7}, \frac{16}{7}\right)$.

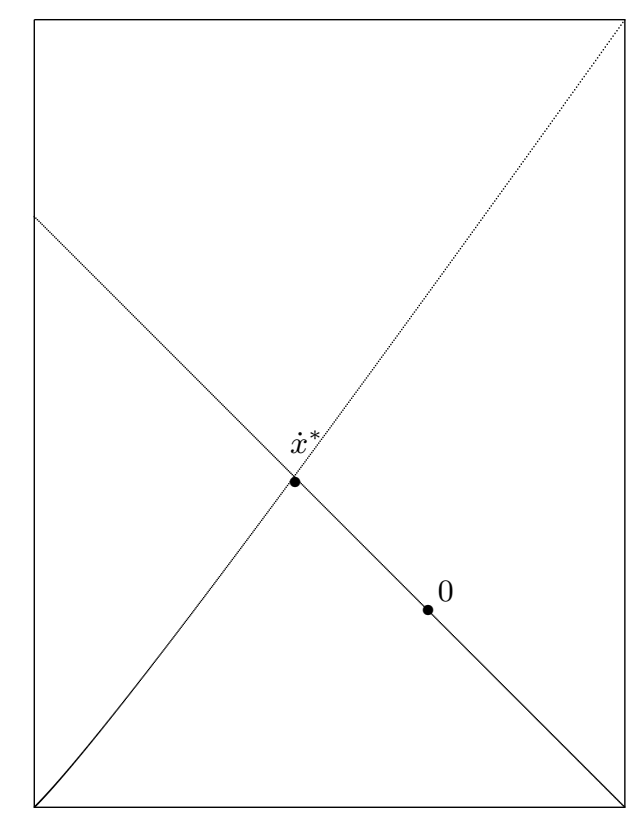

Fig 5 The proportional rule.

Our second restriction concerns the absolute level of prices and the volume of infinitesimal trades. Obviously, there are several ways of choosing $\lambda$ in the proof of Lemma 1. On the other hand, in the same way as a movie can be runned more or less slowly, a trade curve $x(\cdot)$ solving our dynamics can be traced at various speeds: Definition 1 does not convey any "natural" scale for $\dot{x}(t)$ - except that the short-sale constraints imply: $\dot{x}_{i}(t) \geq-\delta_{i}(t)$, every $i$. When $\bar{\mu}(t)>0$, we shall therefore assume that the clearing house maximizes the aggregate volume of trades: $\sum_{i}\left|\dot{x}_{i}^{+}(t)\right|$ - a current practice

\footnotetext{
${ }^{32}$ With the convention $\frac{x}{0}:=0$.
} 
in real-life clearing houses. That this optimization problem admits a (unique) solution is guaranteed by the individual short-sale constraints. This amounts to rescaling the units of a commodity flow, say from ounces to pounds, and taking the available stocks of commodities (whose units is given a priori in the long-run economy $\mathcal{E}$ ) as absolute upper-bounds for this rescaling. ${ }^{33}$ Once this is done, it remains to choose the absolute level of prices so as to maximize $\lambda>0$ under the individual cash-in-advance constraints $p \cdot \dot{x}^{+} \leq \mu_{i}\left(1+\frac{M}{\bar{\mu}}\right)$, every $i{ }^{34}$ A proportional pseudo-flow whose price level has been fixed according to this rule is called a quasi-flow. The example from subsection 4.4. below clarifies how the whole mechanism works.

Definition 2. A monetary flow $(\bar{p}, \dot{x})$ of $T_{z(t)} \mathcal{E}$ is then defined by means of Mertens' algorithm: ${ }^{35}$ select any quasi-flow $(p, \dot{x})$, next start again with the truncated economy restricted to $\left\{c: p_{c}=0\right\}$, as long as this set is non-empty. Since there are finitely many commodities, the algorithm must have an end. Moreover, the price $\bar{p}$, induced by a monetary flow, must belong to $\mathbb{R}_{++}^{C}$. The uniqueness of Mertens' (2003) solution also yields the uniqueness of the monetary flow, at least in terms of trades.

LEMMA 2.- Regardless of the partition of commodities chosen at every step, Mertens' algorithm produces the same final commodity flow $\dot{x}$. Unless $\dot{x}=0$ or $\bar{\mu}=0$, the corresponding price $\bar{p}$ is unique.

Proof of Lemma 2. When $\bar{\mu}>0$ and $\dot{x} \neq 0$ (which means $M>0$ ), the uniqueness of $\dot{x}$ and of the price ratios $(\lambda p)_{\lambda>0}$ follows from Mertens (2003, section 3). The uniqueness of $\tilde{m}_{i}$ follows from (15), and that the vector price $\bar{p}$ from the unique $\lambda$ solving the clearing house's task.

It is worth noticing that, when $\dot{x}=0$, Mertens' algorithm may end up with various price ratios (see Example 2 supra), while, when $\bar{\mu}=0$ and $M>0$, the players' amounts of borowed money, $\tilde{m}_{i} \geq 0$ and the price normalization factor $\lambda>0$ may take various values compatible with $\sum_{i} \tilde{m}_{i}=M$ and $\lambda p \cdot \dot{x}^{+} \leq \tilde{m}_{i}$.

Let $X\left(T_{z(t)} \mathcal{E}\right)$ denote the unique commodity flow of the monetary tangent market $T_{z(t)} \mathcal{E}$, and $P\left(T_{z(t)} \mathcal{E}\right)$ the set of associated prices. Our dynamics is defined by the pair of equations:

$$
\dot{x}(t)=X\left(T_{z(t)} \mathcal{E}\right) \text { and } p(t) \in P\left(T_{z(t)} \mathcal{E}\right) .
$$

It turns out that the mapping $\varphi: z \mapsto X\left(T_{z} \mathcal{E}\right)$ is not continuous in general. This is, of course, partly due to the presence of discontinuities arising from the friction $r(t) .{ }^{36}$

\footnotetext{
${ }^{33}$ To put it differently, the $\delta_{i}(t)$ are given by households' expectations. Therefore, expectations are responsible for the speed of trades or, equivalently, for the liquidity of markets. But the $\delta_{i}(t)$ themselves are bounded by the real stocks $x_{i}(t)$. Thus, it is the stock of commodity in the long-run economy $\mathcal{E}$ that prevents its dynamic state to move at an infinite speed.

${ }^{34}$ When $\bar{\mu}=0$, as already said, we are back either $(M>0)$ to the idealized barter case, where prices really are only ratios among pairs of goods, or $(M=0)$ to no-trade. Consequently, in both cases, price indeterminacy is intrinsic to the model.

${ }^{35}$ See section VIII.A.Def. 5 in Mertens (2003).

${ }^{36}$ There are, however, deeper reasons for this phenomenon, since discontinuity prevails even in the barter case: Firstly, every strategic market game à la Shapley-Shubik involves a discontinuous outcome
} 
Therefore, continuously differentiable curves satisfying (24) do not exist in general, so that we need to rely on a suitable notion of solution. We shall deal with Filippov's concept of solution (see the Appendix, subsection 6.1, as well as Filippov (1988)), which, in essence, consists in replacing $\dot{x}(t)=X\left(T_{z(t)} \mathcal{E}\right)$ by a differential inclusion, where the derivative $\dot{x}(t)$ is only asked to belong to a set of directions lying in a neighborhood of $X\left(T_{z(t)} \mathcal{E}\right)$. This flexibility is key in providing reasonable conditions on the vector field of our dynamics for trade curves to exist. One may be bothered by this technical bend, since differential inclusions hardly provide unique solution curves, while we adopted Mertens'solution concept precisely with the aim of recovering uniqueness. The piece of good news provided by the next section is that this trick shall nevertheless yield generic uniqueness.

\section{Uniqueness and non-neutrality}

This section contains the main results of this paper.

\subsection{Existence}

A monetary policy is continuous (resp. smooth, etc.), when the mapping $\mathbb{R} \ni t \mapsto M_{t}$ is so. Existence of monetary trade curves holds under fairly general conditions, as shown by the next theorem. Observe, in particular, that no interiority assumption is made on the long-run real economy $\mathcal{E}$, nor any Slatter or survival or irreducibility restriction all of them would be at odds with the recent experience of financial markets after the subprime crisis. Similarly, preferences need not be convex. ${ }^{37}$

THEOREM 1.-For every feasible initial state $z(0)$ and for every monetary policy $(M(t))_{t}$ which continuous for a.e. time $t$, then (24) admits a solution curve.

This theorem is somewhat loosely expressed since, so far, we did not spell out the definition of a "solution" to (24) in order not to interrupt too much the body of our analysis. Precise details are given in the Appendix, together with the proof of Theorem 1.

\subsection{Generic nominal uniqueness of trade curves}

Let us endow the space of utilities $u=\left(u_{i}\right)_{i}$ with the sup-norm topology $\left(\left\|u_{i}\right\|=\right.$ $\left.\max _{X_{i}} u_{i}(x)\right)$ and that of continuous monetary policies $t \mapsto M_{t}$ with the topology of

function ; so does every auction as well. Secondy, the Walras correspondence fails to be lower hemicontinuous in general, hence to admit any continuous selection. So does any generalized equilibrium correspondence. Hence, whatever being the viewpoint under which $\varphi$ is analyzed - as a Sapley window's game with limit-orders or a double auction or a generalized equilibrium concept -, it must be discontinuous.

${ }^{37}$ It is even possible to weaken the differentiability assumption on utilities, but we refrain from striving for the utmost generality. 
uniform convergence over compacta. ${ }^{38}$ The next result states that, given aggregate initial endowments in commodities, $\bar{\omega}$, any initial state of outside money $m(0)$, and for a dense subclass of utilities $u$ and monetary policies $M_{t}$, the vector field associated to our dynamics is smooth on an open and dense subset of the feasible set, provided $\bar{\mu}(t)>0$ throughout. The Cauchy-Lipschitz theory of smooth differential equations then implies that, when restricted to this generic subset, the Cauchy problem induced by (24) admits a (piecewise) unique solution path in forward time not only in real but also in nominal terms. In the sequel, $\mathcal{M}^{*}$ denotes the subset of feasible states such that $\bar{\mu}>0$.

THEOREM 2.-Given aggregate initial endowments in commodities, $\bar{\omega} \gg$ 0 , and in outside money $\bar{m}>0$, and for a dense subclass of utilities $u$ verifying the standard smoothness restrictions and smooth policies $M$, the product space $\mathbb{R} \times \mathcal{M}^{*}$ can be partitioned as:

$$
\mathbb{R} \times \mathcal{M}^{*}=\mathcal{R} \cup \mathcal{C}
$$

where $\mathcal{C}$ is the finite, disjoint union of smooth submanifolds, all of them of dimension strictly less than

$$
C(N-1)+N(C+2)+2=\operatorname{dim} \mathcal{R}=\operatorname{dim} \mathbb{R} \times \mathcal{M}^{*} .
$$

Moreover, the restriction of (24) to the (open and dense) subset $\mathcal{R}$ induces a smooth vector field.

In fact, the proof of Theorem 2 contains much more than the statement of the theorem itself says. Rougly speaking, the situation is as follows. First, because expectations exert a non-trivial influence on both the real and the monetary spheres, the space, $\mathcal{M}^{*}$, of allocations in commodities and cash can no more be considered independently of time. They are intrinsically conjugate. Second, for a generic class of parameters of the dynamics, there are large regions within the space-time environment $\mathbb{R} \times \mathcal{M}^{*}$, where local gains-to-trade, expectations and money coexist in a sort of balance, leading to a unique smooth trade path together with a smooth price curve. This balance changes abruptly when we pass beyond the surface of one such region. At best, the change between an inner regime and an outer regime may be marked by a lack of differentiability of the trade and/or the price curve. This is what market makers usually call a "market crunch" (see Fig. 6).

\footnotetext{
${ }^{38}$ That is, the topology over the space of monetary policies $M$ is given by the following family of semi-norms (for $k \in \mathbb{N}, K \subset \mathbb{R}$ compact):

$$
\|M\|_{K}:=\max _{K} M_{t} .
$$
}




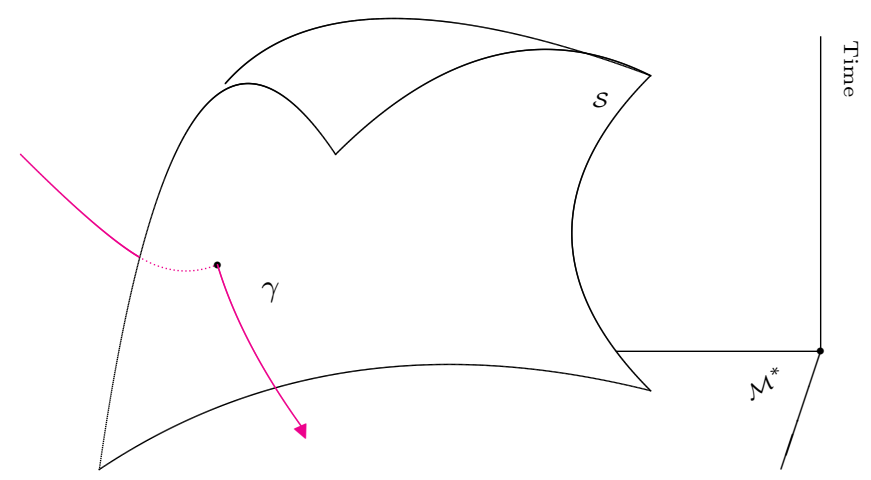

Fig. 6. Crossing a critical boundary may cause a "market crunch".

But reaching the frontier, $\mathcal{S}$, between two regimes, say $\mathcal{D}_{1}$ and $\mathcal{D}_{2}$, may cause a more profond change than simply a "kink" in the trade-and-price curve: if it reaches tangentially the boundary, the curve may quit it (or not) in different directions (see Fig. 7). If it does enter into the new regime, then one nano-second later, of course, a new balance between our three forces will have been recovered, so that the trade-and-price curve will again evolve smoothly in an unambiguous way. But at the very moment we touch a "critical" frontier, various things can happen that cannot be predicted from the past observed in the previous regime.

Notice that this phenomenon already obtains for a barter and myopic economy: ${ }^{39}$ What considerably simplifies the picture in this latter case, however, is that time and space can again be considered separately, while the monetary sector disappears. As a consequence, only the feasible set, $\tau$, of commodity allocationsthe need to be partitionned between "regular" regions and "critical" boundaries. In Example 1 supra (see section 2.1), for instance, there are two regimes, separated by the diagonal, $\theta$, of efficient allocations. For more general economies, generically, there are always finitely many smooth submanifolds playing the role of critical boundaries.

Whether crossing a critical boundary will have no visible effect, or will simply cause a crunch or will result in some momentarily unpredictable behavior can be easily checked in the barter-myopic case. Indeed, according to Filippov's theory of discontinuous vector fields, one simply need consider the following possibilities (see Fig. 7):

(a) if the vector field arising from the inner regime points in the direction of the outer regime, where the vector field flows away from the critical submanifold $\mathcal{S}$, then the trade path continues its motion in the outer regime (possibly with some kink);

(b) if both the vector field of the inner regime $\mathcal{D}_{1}$ and that of the outer regime $\mathcal{D}_{2}$ flow into $\mathcal{S}$, then after having reached $\mathcal{S}$, the solution curve will stay in $\mathcal{S}$ and possibly slide along the boundary of the two regimes. The latter kind of trajectory is called a sliding motion. Notice that forward uniqueness is still preserved in this case. Notice as well that, whenever the two vector fields arising from $\mathcal{D}_{1}$ and $\mathcal{D}_{2}$ are exactly opposite (in the sense that $\dot{x}^{+}+\dot{x}^{-}=0$ where $\dot{x}^{+}$is the limit of $\dot{x}(t)$ as $x(t)$ reaches $\mathcal{S}$ from $\mathcal{D}_{1}$, and $\dot{x}^{-}(t)$ the same limit for $x(t)$ reaching $\mathcal{S}$ from $\left.\mathcal{D}_{2}\right)$, then they cancel each other at $\mathcal{S}$ : the

\footnotetext{
${ }^{39}$ See Giraud (2004).
} 
point $x^{*}$ at which $x(t)$ reaches $\mathcal{S}$ from both sides is a rest-point. In this case, the motion does not slide along $\mathcal{S}$. This is exactly what occurs along the critical submanifolds whose union builds $\Theta$.

Finally, (c) if the vector field arising from $\mathcal{D}_{1}$ is tangent to $\mathcal{S}$ while the vector field in $\mathcal{D}_{2}$ flows away from $\mathcal{S}$, then either the path leaves the boundary immediately in order to enter into $\mathcal{D}_{2}$, or there exist (Filippov) solutions that reach the boundary and stay in it afterwards, for a time that cannot be deduced from the inner regime. In this latter case (only), forward uniqueness is lost.

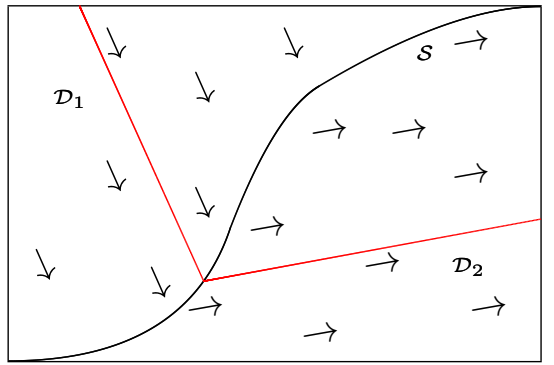

(a)

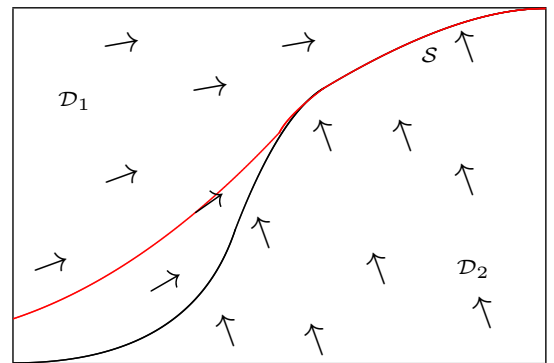

(b)

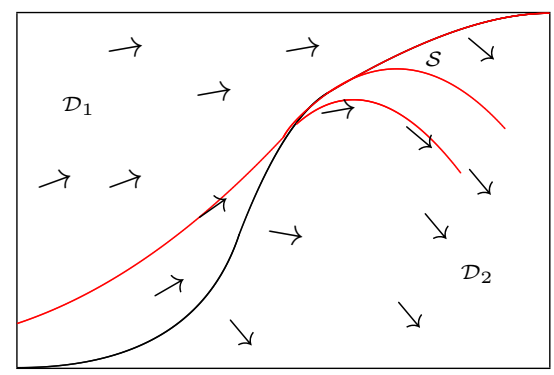

(c)

Fig. 7. The dynamical systems are smooth on $\mathcal{D}_{1}$ and $\mathcal{D}_{2}$, and disctontinuous at $\mathcal{S}$.

Adding the monetary field while keeping the myopia restriction does not tremendously alter this relatively simple picture: it only enlarges the space, $\tau$, of commodity allocations into the space $\tau \times \mathbb{R}^{N}$ of commodity and monetary allocations. Adding expectations drastically modifies the situation. It enlarges again the space under scrutiny into $\mathcal{M}^{*}$ by taking into account the households' saving behavior. On the other hand, the boundary between two regimes may, now, be in time and not solely in space. One regime may represent the dynamical state of the world up to a given time and the other regime the state after that time. When the change of regime occurs solely "in time", then we can be sure that it is entirely due to a shock in expectations. Otherwise, of course, the situation is more complicated. What Theorem 2 says, in essence, is this: expectations, real gains-to-trade and money will interact with each other in creating in the space-time environment $\mathbb{R} \times \mathcal{M}^{*}$ a finite number of smooth manifolds that will play the role of critical boundaries. 


\subsubsection{How "rational" are locally rational expectations?}

We already know from Lemma 1 that, together with the individual maximization of short-run utility, locally rational expectations imply that the no-default condition be satisfied as an equality (16), i.e., that no household ever goes bankrupt: $p \cdot \dot{x}_{i}=0$ a.e. $i$. As a by-product of Theorem 2, we can add a second feature formalizing the sense in which expectations in this paper are "locally rational". Indeed, the piecewise smoothness of trade curves enables us to state the following version of gross-substitutability in tangent markets: ${ }^{40}$

Proposition 3.- Under the standard smoothness restrictions, and for every regular state $z(t) \in \mathcal{R}$ such that $\delta_{i}(t) \gg 0$ for every $i$, one has:

$$
\frac{\partial}{\partial \delta_{i}^{c}(t)} P\left(T_{z(t)} \mathcal{E}\right) \leq 0 \quad \text { for every commodity } c .
$$

Suppose that trader $i$ anticipates the price of commodity $c$ to increase in the near future. If he is positively endowed with good $c$, he should save some additional amout of this very commodity in order to sell it latter on. Hence, $\delta_{i}^{c}(t)$ should decrease. Proposition 3 says that, consequently, the current price $p_{c}(t)$ will rise (or, at least, will not decline) if everything else is being kept fixed. Conversely, if $i$ expects $p_{c}(t)$ to decrease, hence decides to sell more of commodity $c$ on the tangent market $T_{z(t)} E$, then $p_{c}(t)$ will decrease. Hence, $i$ 's expectations turn out to be always self-fulfilled in the short-run along a trade curve. Notice, of course, that consumers $i$ and $j$ may have contradictory expectations over the same commodity $c$ at the same time $t$. In this case, only the trader whose saving behavior was most influential on the resulting price will see his viewpoint confirmed by the market.

\subsection{Stability and money non-neutrality}

We now address two questions that turn out to be intimately related: How stable are stationary points of our dynamics ? and: Is money neutral in our model ? It is clear that, if both $m$ and $M$ are multiplied by some constant $\lambda>0$, then nothing changes in the analysis. This means that there is no money illusion. However, if $m$ and $M$ are changed disproportionately, then there will typically be a change in the long-run real variables characterizing the monetary trade curves of the economy. We show in this subsection how to characterize the short-run and long-run impact of such a monetary change on the real sector. Unless otherwise specified, we assume hereafter that $\bar{m}>0$.

\footnotetext{
${ }^{40}$ One could drop the regularity restriction on the state $z(t)$ at the cost of replacing the differential operator by Clarke's generalized gradient, as in Bonnisseau et alii (2001). We do not stop for details.
} 


\subsubsection{Local gains-to-trade}

We now formally define the measure $\gamma(x)$ of local gains-to-trade first alluded to in section 2.1 above.

Let $\dot{z}_{i} \in \mathbb{R}^{C}$ be an infinitesimal trade vector of $i$ in some tangent market, with positive component representing purchases and negative ones representing sales. For any scalar $\gamma \geq 0$, define:

$$
\dot{z}_{i}^{c}(\gamma):=\min \left\{\dot{z}_{i}^{c}, \frac{\dot{z}_{i}^{c}}{1+\gamma}\right\}
$$

The vector $\dot{z}_{i}(\gamma)$ entails a diminution of purchases in $\dot{z}_{i}$ by the fraction $1 /(1+\gamma)$. There are local gains to $\gamma$-diminished trades in the barter tangent market $T_{\delta} \mathcal{E}$ if there exist feasible infinitesimal trades $\left(\dot{z}_{i}\right)_{i}$ such that $\dot{z}_{i} \geq \delta_{i}$ for all $i$, and $v_{i}\left(\dot{z}_{i}(\gamma)\right) \geq 0$ for all $i$ with at least one strict inequality. In words, it should be possible for households to Pareto-improve on no-trade in spite of the $\gamma$-handicap on trades. For every $x \in \tau$, the measure $\gamma(x)$ is the supremum of all handicaps that permit Pareto-improvement. Clearly, $x$ is Pareto-optimal if, and only if, $\gamma(x)=0$. An important property of the measure $\gamma(\cdot)$ is that it is invariant with respect to rescaling the units of commodities. ${ }^{41}$

The monetary version of $\gamma(\cdot)$ goes as follows: (i) If $\bar{\mu}>0$, then consider the linear economy obtained by ignoring, in $T_{z} \mathcal{E}$, those traders $i$ with $\mu_{i}=0$. Denote by $\gamma^{*}(\delta)$ the measure applied on the short-sale constraints of those traders who are positively endowed with money. Local gains-to-trade are then defined as: $\gamma(z):=\gamma^{*}(\delta)$. (ii) If $\bar{\mu}=0$, then $\gamma(z):=\gamma(\delta)$.

The next Proposition is the key result for Theorem 3 to follow, but is also interesting in its own right.

Proposition 4.-(i) Suppose that, for every $i$, whenever defined, $\nabla u_{i}(x)>$ $>0$. For every feasible $z$, if the monetary flow of $T_{z} \mathcal{E}$ verifies $\dot{x}=0$, then one of the following must be true:

$$
\begin{aligned}
& \text { (a) Either } \gamma(z)=0 \text {, } \\
& \text { (b) or } r>\gamma(z) \text {, } \\
& \text { (c) else, } \delta=0 .
\end{aligned}
$$

(ii) If $r>\gamma(z)$, the unique monetary flow is no-trade.

Proposition 4 (i) tells us under which conditions no trade will occur on a tangent market. Three situations may be identified: Either (a) investors do not believe that there are gains to trade - that is, the current allocation of commodities put on the market as a result of people's expectations is already Pareto-optimal. Or (b) the cost of borrowing

\footnotetext{
${ }^{41}$ This follows, e.g., from Theorem 1 in Dubey \& Geanakoplos (2003a). As a consequence, $\gamma(x)$ is not affected by the procedure applied by our clearing house in order to fix trades and prices.
} 
inside money is too heavy in both countries $k=1$, 2in comparison with current gains-totrade as envisaged by people -i.e., the cash-in-advance constraint prevents from trading people who, otherwise, would be willing to do so. Else (c), investors are so pessimistic that they refuse to trade and save all their commodities. Proposition 4 (ii) provides a partial converse: If the current interest rate is above the threshold provided by current gains-to-trade (given households' savings) in both countries, then no-trade must occur.

\subsubsection{Asymptotic local efficiency}

A feasible commodity allocation $x \in \tau$ is locally Pareto-optimal if there does not exist any $\mathcal{C}^{1}$ path $\phi:[a, b) \rightarrow \tau$ of monetary trades such that $\phi(a)=x$ and $\nabla u_{i}\left(x_{i}\right) \cdot \dot{\phi}(x) \geq 0$ for every $i$, with at least one strict inequality. Let us denote by $\theta$ the set of such allocations. Clearly, $x \in \theta$ if, and only if, 0 is Pareto-optimal in $T_{x, \delta, \mu, m, M} \mathcal{E}$, whatever being the variables $(\delta, \mu, m, M)$. On the other hand, it suffices that utilities be weakly quasi-concave (at least on a neighborhood of $\theta$ ) for every locally optimal allocation to be globally efficient. For any scalar $\gamma>0$, let us also denote by $\theta^{\gamma}$ the set of allocations $x \in \theta$ for which there are no local gains to $\gamma$-diminished trades.

In what follows, given some trajectory $\varphi(\cdot)$ in $\mathcal{M} \times \mathbb{R}$, we may consider its restriction, $x(\cdot)$, over $\tau$ - which we call a trade curve. A point $x \in \tau$ is said to be stationary whenever the constant mapping $\equiv x$ is a (degenerate) trade curve of the dynamics. A feasible allocation $x$ is a limit-point of a curve $x(\cdot):[a, b) \rightarrow \tau$ if there exists a sequence $\left(t_{n}\right)_{n}$ tending to $+\infty$ such that $x\left(t_{n}\right) \rightarrow x$. Let $\Omega(x(\cdot))$ denote the subset of limit-points of $x(\cdot)$. A point $x \in \tau$ is locally stable if every solution curve that does not start too far away from $x$ remains in a neighborhood of it.

We are now ready to state the central result of this paper.

THEOREM 3.-Under the standard smoothness assumptions and for every feasible initial state $z(0) \gg 0$,

(i) Suppose that the two following conditions are satisfied:

(a) Expectations are such that, for every $i, x_{i}^{c}(t)>0 \Rightarrow \delta_{i}^{c}(t)>0$ for every $c$, and almost every $t$;

(b) $t \mapsto M(t)$ grows sufficiently rapidly, so that:

$$
M(t) \geq \frac{\bar{\mu}(t)}{\gamma(z(t))}, \quad \text { a.e. } t
$$

then every trade curve converges to some point $x^{*} \in \theta$.

(ii) On the contrary, if the length of time where markets are not sufficiently liquid verifies ${ }^{42}$

\footnotetext{
${ }^{42}$ Here, $\lambda(\cdot)$ is the Lebesgue measure.
} 


$$
\lambda\left[\left\{t: \gamma(z(t))<\frac{\bar{\mu}(t)}{M(t)}=r(t)\right\} \cap\{t: \bar{\mu}(t)>0\}\right]>0,
$$

then, at some time $t^{*}$, the flow of $T_{z\left(t^{*}\right)} \mathcal{E}$ coincides with no-trade whatever being the investors' expectations, and the state will rest on $x\left(t^{*}\right)$ as long as the ratio of inside money to outside available cash does not increase sufficiently so as to verify (27) for a subset of time $t \geq t^{*}$ of positive measure.

(iii) Every locally optimal allocation $x \in \theta$ is a locally stable stationary point.

(iv) Consider two monetary policies $M_{1}(\cdot)$ and $M_{2}(\cdot)$ applied on the same economy $\mathcal{E}$, such that, on the time interval $\left[t_{a}, t_{b}\right]$, traders entertain the same expectations (i.e., the restrictions of $\mu_{i}(\cdot), \sigma_{i}(\cdot)$ to $\left[t_{a}, t_{b}\right]$ are a.e. identical for every $i$ ), and such that

$$
\gamma(z(t))>\frac{\bar{\mu}(t)}{M_{k}(t)}=r_{k}(t), \quad k=1,2
$$

for a.e. $t$ in $\left[t_{a}, t_{b}\right]$. Then, the trade curves $x_{k}(\cdot)$ followed in $\tau$ during this time interval $\left[t_{a}, t_{b}\right]$ are identical. Only prices $p(\cdot)$ depend upon the chosen monetary policy.

A couple of remarks are in order.

1) That a condition akin to (27) is necessary for escaping from any liquidity trap should be made clear by the following example. Consider the two-good real economy $\mathcal{E}:=\left(u_{1}, u_{2}, \omega_{1}, \omega_{2}\right)$ with $u_{1}(x)=x_{1}, u_{2}(x)=x_{2}, \omega_{1}=(1,2), \omega_{2}=(3,3)$. Obviously there are still gains-to-trade at $\omega$ (i.e., $\gamma(\omega)>0$ ). Suppose, however, that expectations are such that $\delta_{1}(0)=(1,0)$ and $\delta_{2}(0)=(2,3)$. The tangent market $T_{z(0)} \mathcal{E}$ reduces to the linear economy of Fig. 4 above. Hence, whatever being the amount of inside money injected, the economy remains trapped at $\omega$.

Conversely, in $(29), \gamma(z(t))<r(t)$ will open a liquidity trap only whenever, at the same time, $\bar{\mu}(t)>0$. Otherwise, we know indeed that the monetary flow degenerates to some Walras equilibrium which becomes independent from the monetary sector.

2) (iii) states that each locally Pareto-optimal allocation in voluntary trades is locally stable. Given the local shape of $\theta$ (a smooth submanifold of dimension $C-1$ whenever $\mathcal{E}$ fulfills the standard smoothness restrictions), it is impossible to improve this property to local asymptotic stability: the later would require that every curve $x(\cdot)$ starting not too far avay from $x$ should converge to it. But this is impossible if $x(\cdot)$ starts at $x(0) \in \theta$ near $x$. This drawback (lack of asymptotic stability) is not peculiar to the our dynamics, but inherent to every non-tâtonnement approach.

3) Most textbooks devoted to monetary theories with rational expectations conclude that money is non-neutral in the short-run, but neutral in the long-run. Here, we do not get this conclusion for the following reason: in the short-run, if $\gamma(x) \neq r$, a sufficiently small change in $r$ will not affect the direction in which the state of the economy moves. 
Indeed, either $\gamma(x)<r$, in which case there is no trade (part (ii) of Theorem 3); or $\gamma(x)>r$, in which case the long-run economy moves in the same direction, whatever being the monetary policy (part (iii)). In this narrow sense, money can be said to be locally neutral in the short-run - "locally" because the preceding argument holds only for "small" changes in the monetary variables $r$ and $M$. Observe, nevertheless, that if $\gamma(x)=r$ (a non-generic event), then the slightest change of $r$ will have a real effect, even in the short-run.

4) Now, in the long-run, different amounts of inside or outside money will induce different trade curves in real terms. Indeed, if $r$ is fixed, then the trajectory followed by the long-run economy will stop at some point $x \in \tau$ where $\gamma(x)=r$. If $r \neq r^{\prime}$, then $x \neq x^{\prime}$. As a consequence, money is non-neutral in the long-run.

5) In the literature just alluded to, one often asks whether money is super-neutral, meaning that a change in the growth of the level of money would not affect real variables. It should be clear from the preceding discussion that money is not super-neutral in our model since there is a minimal rate of growth for inside money, below which the economy remains traped in a liquidity trap before having reached an optimum.

6) Observe that, in most of the literature derived from Lucas (1972), information is asymmetric, and it is an unanticipated change in the money level that makes money non-neutral. On the other hand, in such models, there is usually no outside money. As a consequence, when information becomes symmetric, money non-neutrality reduces to money illusion in this approach. By contrast, here as in Dubey \& Geanakoplos (2003a), the presence of both outside and inside money enables to combine no money illusion (the whole dynamics is 0 -homogeneous with respect to $(m, M)$ ) with money non-neutrality).

7) The sense in which (27) yields an optimal growth rate of inside money is provided by (iii). Indeed, the later says that, as long as (29) holds, a further increase of $M(\cdot)$ will have no real effect. According to our local quantity theory of money (20), it must therefore induce inflation. We shall see this phenomenon at work through the next example.

\subsection{An example}

Before plunging into an example, let us make a simple observation. ${ }^{43}$

LEMmA 3.- Taken together, the cash-in-advance constraint, $p(t) \cdot x_{i}^{+}(t) \leq$ $\tilde{m}_{i}(t)+\mu_{i}(t)$, and the no-default condition conveniently rewritten as:

$$
(1+r(t)) \tilde{m}(t) \leq p(t) \cdot \dot{x}_{i}^{-}(t)+\left[\mu_{i}(t)+\tilde{m}_{i}(t)-p(t) \cdot x_{i}^{+}(t)\right]^{+}
$$

are equivalent with the following non-linear budget constraint:

$$
\langle p(t), \dot{x}\rangle_{r(t)}:=p(t) \cdot x_{i}^{+}(t)-\frac{1}{1+r(t)} p(t) \cdot x_{i}^{-}(t) \leq \mu_{i}(t) .
$$

\footnotetext{
${ }^{43}$ This is a reformulation of Lemma 1 in Dubey \& Geanakoplos (2006a).
} 
Proof of Lemma 3. Take any infinitesimal trade $\dot{x}_{i}(t)$ verifying (30) and set $\tilde{m}_{i}(t):=$ $\left[p(t) \cdot \dot{x}_{i}^{+}(t)-\mu_{i}(t)\right]^{+}$. Then the cash-in-advance constraint is easily checked, while $\tilde{m}_{i}(t)$ is either zero or fulfills:

$$
\begin{aligned}
\tilde{m}_{i}(t) & =p(t) \cdot \dot{x}_{i}^{+}(t)-\frac{1}{1+r(t)} p(t) \cdot \dot{x}_{i}^{-}(t)-\mu_{i}(t)+\frac{1}{1+r(t)} p(t) \cdot \dot{x}_{i}^{-}(t) \\
& \leq \frac{1}{1+r(t)} p(t) \cdot \dot{x}_{i}^{-}(t) \quad \text { because of }(30)
\end{aligned}
$$

so that the no-default condition is satisfied as well. Conversely, suppose that $\dot{x}_{i}(t)$ verifies the two above mentioned constraints. Then,

$$
\frac{-1}{1+r(t)} p(t) \cdot \dot{x}_{i}^{-}(t) \leq \frac{1}{1+r(t)}\left(\mu_{i}(t)-p(t) \cdot \dot{x}_{i}(t)-r(t) \tilde{m}_{i}(t)\right) .
$$

Hence:

$$
\begin{aligned}
\left\langle p(t), \dot{x}_{i}(t)\right\rangle_{r(t)} & \leq \frac{r(t)}{1+r(t)} p(t) \cdot \dot{x}_{i}^{+}(t)+\frac{1}{1+r(t)} \mu_{i}(t)-\frac{r(t)}{1+r(t)} \tilde{m}_{i}(t) \\
& \leq \mu_{i}(t) \quad \text { because of the cash-in-advance constraint. }
\end{aligned}
$$

This observation provides us with an alternate characterization of monetary pseudoflows in case $\bar{\mu}(t)>0$. The optimization programme of player $i$ in $T_{z} \mathcal{E}$ consists indeed in maximizing his short-run utility $v_{i}$ under the budget constraint (30) induced by the non-linear pricing rule $\langle\cdot, \cdot\rangle_{r(t)}$. Whenever $r(t)=0$, this reduces to the standard inner product.

Suppose, now, there are two agents and two commodities $(N=C=2) \cdot{ }^{44}$ Shortsale constraints at time $t$ are given by $\delta^{1}(t)=\delta^{2}(t)=(50,50)$; private outside cash available for trade is $\mu^{1}(t)=\mu^{2}(t)=€ 5$; inside money is $M(t)=€ 90$. Finally, shortrun marginal utilities are $v^{1}\left(\dot{x}_{1}^{1}, \dot{x}_{2}^{1}\right)=\frac{10}{75} \dot{x}_{1}^{1}+\frac{3}{25} \dot{x}_{2}^{1}$, and $v^{2}\left(\dot{x}_{1}^{2}, \dot{x}_{2}^{2}\right)=\frac{3}{25} \dot{x}_{1}^{2}+\frac{10}{75} \dot{x}_{2}^{2}$. At the unique monetary flow, prices are $p_{1}(t)=p_{2}(t)=1$; interest rate is $r(t)=\frac{1}{9}$; infinitesimal trades verify $\dot{x}^{1}(t)=(50,-50), \dot{x}^{2}(t)=(-50,50)$. Household 1 goes short in commodity 2 and buys 1. For this purpose, it spends his $€ 5$ and buys 5 units of good 1. It also borrows $\tilde{m}_{1}(t)=€ 45$ from the Bank, promising to repay $(1+r(t)) \tilde{m}_{1}(t)=€ 50$. This loan is spent to buy 45 additional units of good 1. Finally, agent 1 sells 50 units of good 2 to agent 2, and is able to repay the Bank. Traders' final gradients are not parallel, because:

$$
\frac{\frac{\partial v^{1}}{\partial x_{1}^{1}}\left(\dot{x}^{1}(t)\right)}{p_{1}(t)}=(1+r(t)) \frac{\frac{\partial v^{1}}{\partial x_{2}^{1}}\left(\dot{x}^{1}(t)\right)}{p_{2}(t)} \quad \text { and } \quad \frac{\frac{\partial v^{2}}{\partial x_{2}^{2}}\left(\dot{x}^{2}(t)\right)}{p_{2}(t)}=(1+r(t)) \frac{\frac{\partial v^{2}}{\partial x_{1}^{2}}\left(\dot{x}^{2}(t)\right)}{p_{1}(t)} .
$$

\footnotetext{
${ }^{44}$ In order to facilitate comparisons, we adopt a linearized version of Dubey \& Geanakoplos (2003a)'s example (see their section 6).
} 
This misalignment is clearly due to the transaction cost $r(t)$, and confirms that a flow may fail to be Pareto-optimal in the tangent market. ${ }^{45}$

In order to understand how the clearing-house works according to our second rectriction, observe that a continuum of alternate pseudo-flows would have been conceivable. For instance, $p_{1}(t)=p_{2}(t)=2 ; r(t)=\frac{1}{9}$ (unchanged), and $\dot{x}^{1}(t)=(25,-25), \dot{x}^{2}(t)=$ $(-25,25)$ is a pseudo-flow of the tangent market (see Fig. 8).

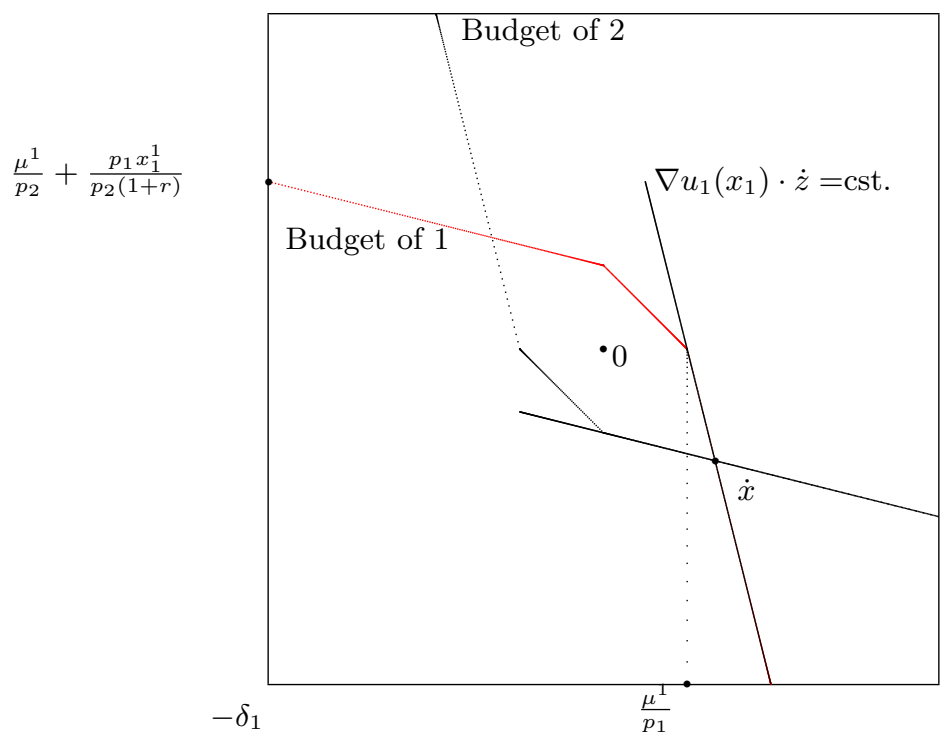

Fig. 8. An interior pseudo-flow

In Fig. 8 above, the red segments figure the frontier of player 1's budget set. (we have dropped the time index in order to shorten notations.) Similarly, every point in the segment joining the origin (no-trade) to the unique monetary flow (bottom-right corner) can be turned into a pseudo-flow by choosing the appropriate price level. Obviously, however, only the bottom-right corner does maximize the volume of infinitesimal trades. Not surprisingly, it also minimizes the price level.

What happens, now, if, everything else being kept fixed, $M(t)$ decreases ? Then $r(t)$ increases above $\gamma(z(t))=\frac{1}{9}$, and the unique flow is no-trade (Proposition 4 (ii)). On the contrary, when $M(t)$ increases, $r(t)$ decreases below $\gamma(z(t))$. Clearly, real trades cannot change. How do prices evolve as $M(t)$ increases? For a given $r(t)<\frac{1}{9}$, one gets: $p_{1}(t)=p_{2}(t)=\frac{1+r(t)}{10 r(t)}$. Therefore, as soon as $M(t)>\gamma(z(t)) / \bar{\mu}(t)$, then the "classical dichotomy" holds in the short-run: an increase of inside money just increases prices proportionally at time $t$ and decreases the interest rate without affecting real trades (and even without affecting the speed of trades). Thus, above a certain finite threshold,

\footnotetext{
${ }^{45}$ Observe that a monetary flow need not be Pareto-optimal within the tangent market even when $r=0$, as shown by Example 2 above, where no-trade is the unique flow although it is not efficient. Of course, when $r=0, \mu_{i}>0, \delta_{i} \gg 0$ and $\nabla u_{i}\left(x_{i}\right) \gg 0$ for every $i$, every flow is a first-order flow which is Pareto-optimal with respect to short-run utilities.
} 
an increase of inside money has no impact but nominal inflation.

Suppose, now, that $M(t)$ is fixed. What happens as $\bar{\mu}(t)$ varies due, say, to some change in households'expectations or to some fiscal policy ? For simplicity, let's restrict ourselves to proportional variations of cash for each household. Whenever $\bar{\mu}(t)>$ $M(t) \gamma(z(t))$, no-trade is the unique flow and prices are indeterminate. If $\bar{\mu}(t)=M(t) \gamma(z(t))$, the state of the long-run economy is driven by the unique monetary flow of its tangent market. When $\bar{\mu}(t)$ further decreases, the economy moves in the same direction and at the same spped, but $r(t)$ decreases and prices decrease as well.

One can summarize the short-run effects of (i) monetary policy $(M(t)$ varies) and (ii) non-discriminatory fiscal policy $\left(\mu_{i}(t)\right.$ varies proportionally for each household $\left.i\right)$ by means of the following two diagrams:

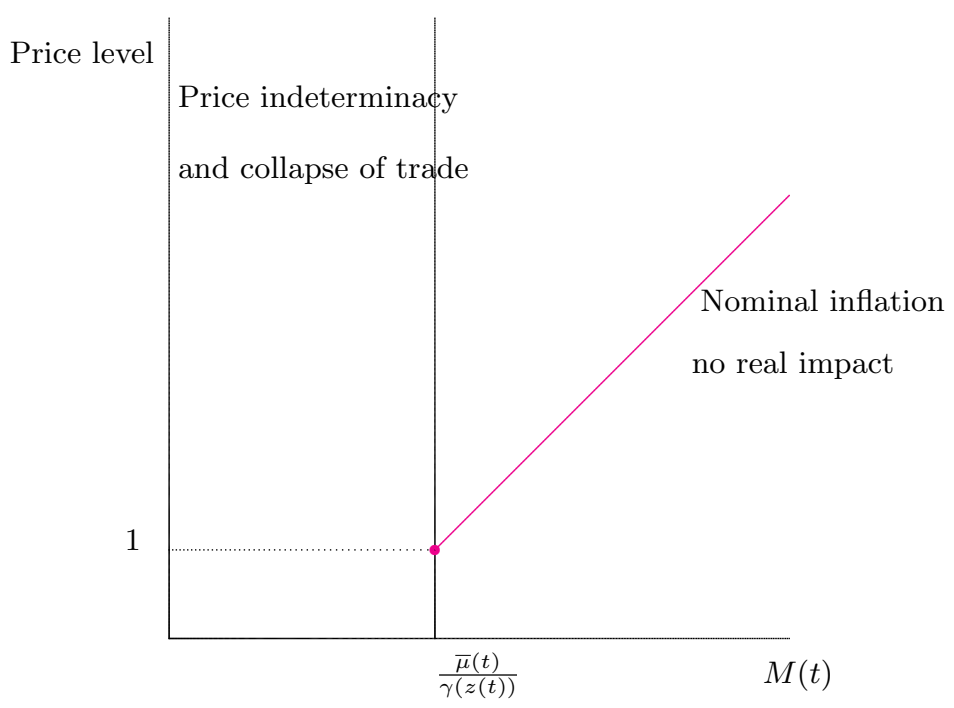

Fig. 9. Outside cash, $\mu(t)$, fixed; $M(t)$ varies. 


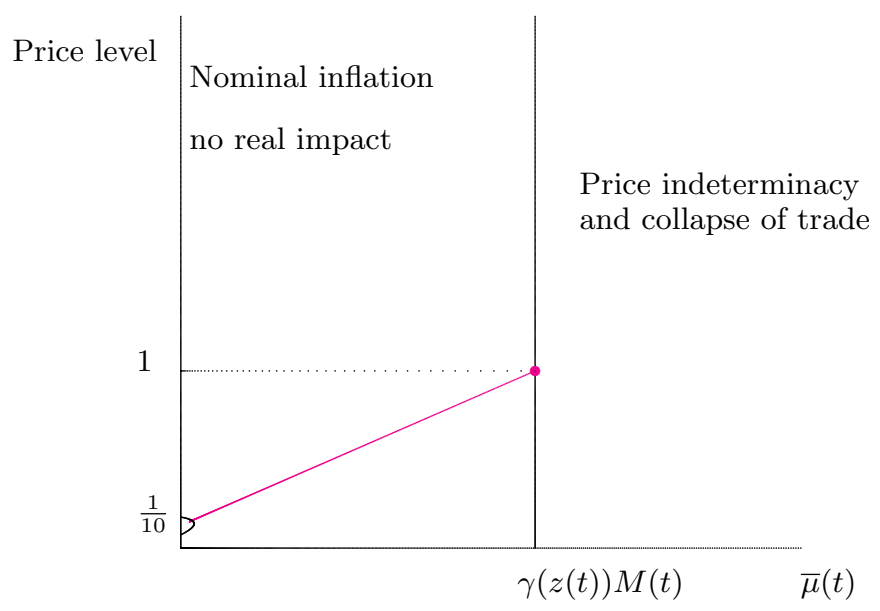

Fig. 10. Inside money, $M(t)$, fixed; $\bar{\mu}(t)$ varies.

When compared with Fig. 6 in Dubey \& Geanakoplos (2003a), here, there is no "hyperinflation phenomenon" due to purely monetary factors: As $M(t)$ decreases to $\bar{\mu}(t) / \gamma(z(t))$ $(\bar{\mu}(t)$ being fixed), both prices converge to 1 . At the moment where $M(t)<\bar{\mu}(t) / \gamma(z(t))$, prices are indeterminate. Similarly, when $M(t)$ is fixed, as $\bar{\mu}(t)$ increases towards $\gamma(z(t)) M(t)$, prices converge towards $(1,1)$ and then are indeterminate. Of course, the linear tangent market of this example can be approximated by a strictly concave one by replacing each linear short-run preference $v_{i}$ by $v_{i}+\varepsilon \sum_{c} \sqrt{x_{c}^{i}}$. One then sees that our diagrams are degenerate limits of Fig. 6 and 7 of Dubey \& Geanakoplos (2003a).

Our analysis, however, has the feature that, whenever all households' expectations are so pessimistic that $\delta_{i}(t)$ decrease, then prices rise. Indeed, suppose, to simplify the picture, that both households in the preceding example share "isomorphic" expectations, so that $\delta_{1}(t)=\delta_{2}(t) \rightarrow 0^{+}$. Then, the Edgeworth box will reduce to its central point: the speed of trades declines to zero, while the price level rises much faster than linearly since it reaches infinity over a finite move of $\delta_{i}(t)$. This explosion of price can be called hyperinflation. Thus, in this model, hyperinflation occurs only when households lose so much confidence in money that they even don't want to sell commodities for money any more. And in such a case, the monetary policy is irrelevant in order to prevent such a phenomenon!

Going back to the doctrinal debate already alluded to, our analysis conjugates Friedman's remodeling of the QTM into a theory of the demand for money together with the Keynesian money non-neutrality. Arguing that prices would fall in a depression, thereby raising the purchasing power of wealth held in unspent money, many monetarists claim indeed that the price-induced rise in the real value of cash balances would then stimulate spending directly. It follows that a rise in the real balances and hence spending could be accomplished just as easily via a monetary expansion, validating the potency of monetary policy even in a depression. Here, this argument holds partially, provided households' expectations do not affect their willingness to sell commodities (i.e., $\delta_{i}(t)$ ). 
But even then, it holds only partially since, if an economy is located in a liquidity trap, a rise in the quantity of money $M(\cdot)$ (due to monetary policy) or in $m_{i}(t)$ (due to fiscal policy) does not have any effect as long as (27) does not hold (in particular, as long as it does not influence $\mu_{i}(t)$ positively). Nevertheless, both policies may be operative if they succeed in enforcing (27) for a time interval of positive length.

Looking now at the dynamic picture, one sees that the trade curve followed by our long-run economy depends upon the quantity of circulating money in the following way:

$(\alpha)$ either $\mu(t) \gg 0$ (that is, traders' expectations are not too pessimistic) and there is enough inside money throughout, in which case the economy follows a unique trade curve $\gamma$ (which coincides, here, with the non-monetary "Walrasian" trade curve); in particular, it converges to some Pareto-optimal point, $r(t) \rightarrow 0^{+}$and prices remain bounded ;

$(\beta)$ or there is not enough inside money, i.e., $0<M(t)<\frac{\bar{\mu}(t)}{\gamma(z(t))}$ during a nonnegligible interval of time $\left[t_{a}, t_{b}\right]$. In this case, the economy stops at $x\left(t_{a}\right)$ (even though $\left.x\left(t_{a}\right) \notin \theta\right)$, with $r\left(t_{a}\right)>0$;

$(\gamma)$ or there is not enough outside money. For instance, one of the two traders $i$ become so pessimistic that $\mu_{i}(t)=0$ (while $\mu_{j}(t)>0$ ) during a non-negligible interval of time $\left[t_{a}, t_{b}\right]$. Then, again, the economy stops at $x\left(t_{a}\right)$, whatever being the amount of outside money $M\left(t_{a}\right)$ injected in the system (player $j$ has no counterpart with whom she could trade).

$(\delta)$ or there are not sufficiently many commodities offered for sale, i.e., $\delta_{i}(t) \rightarrow 0^{+}$ for at least one of the two households.

\section{A double-auction with limit-price orders}

As we have just seen, our dynamics results in a line $\gamma(\cdot)$ in $\mathcal{M} \times \mathbb{R}$ (possibly degenerate at some point whenever the economy remains bloked in a liquidity trap). Imagine, now, an electron microscope aimed at a point $z \in \mathcal{M}$. Under enlargement, the neighborhood of $z$ and the environment above it become linear: we get the tangent market $T_{z} \mathcal{E}$. With even greater magnification, we see, instead of the reduced-form model summed up in section 2.3 , a strategic market-game $G\left[T_{z} \mathcal{E}\right]$, where investors send limit-price orders in continuous time to a central clearing house which instantaneously execute some of them according to some rules that we describe now. ${ }^{46}$ From the standpoint of the central clearing house, investors are "invisible": all it can see is the order book populated by a myriad of anonymous orders.

Recall that each agent $i$ actually stands for a continuum, [0,1], of identical clones, having the same utility, $u_{i}(\cdot)$, the same initial endowment, $\omega_{i}$, and the same saving

\footnotetext{
${ }^{46}$ In essence, we adapt Merten's (2003) limit price mechanism to Dubey \& Geanakolos (2003) monetary setting. The stragegic mechanism can be viewed alternatively as the multi-item extension of double auction, or as the extension of Shapley's windows model (see Sahi \& Yao (1989)) to limit-price orders. See Giraud (2003) for an introduction.
} 
functions $s_{i}(\cdot), \sigma_{i}(\cdot)$, so that the space of players is $[0,1]^{N}$ equipped with the product Lebesgue measure $\lambda_{\mid[0,1]}^{\otimes N}=: \nu$.

\subsection{The barter case once again}

For simplicity, let's begin with a two-good barter economy.

\section{Strategies as fictitious agents}

A strategy of player $i$ in the local game $G\left[T_{z} \mathcal{E}\right]$ associated to the tangent market $T_{z} \mathcal{E}$ is to send a limit-price order to the market. Only selling orders are allowed — but this implies no loss of generality: if a player wants to buy a commodity, he just has to sell money. A limit-order to sell item commodity $\ell$ in exchange for item $c$ gives a quantity $q_{\ell}$ to be sold, and a relative price $p_{\ell}^{+} / p_{c}^{+}$. The order is to sell up to $q_{\ell c}$ units of item $\ell$ in exchange for item $c$ if the actual relative price verifies $p_{\ell} / p_{c} \geq p_{\ell}^{+} / p_{c}^{+}$. The amount $q_{\ell}$ put up for sale will stay untouched at any price $p_{\ell}<p_{c} p_{\ell}^{+} / p_{c}^{+}$, and is intended to be fully sold at any price $p_{\ell} \geq p_{c} p_{\ell}^{+} / p_{c}^{+}$. When $\frac{p_{\ell}^{+}}{p_{c}^{+}}=0$, one gets a familiar market order. A limit-order to "sell" commodity $\ell$ against $c$, at relative prices $p_{c}^{+}=0, p_{\ell}^{+}>0$ is, in fact, an order not to buy $c$, and to sell as much of $\ell$ as possible. We now recall Mertens' trick. The key in understanding the relationship between the reduced-form model and $G\left[T_{z} \mathcal{E}\right]$ lies in Mertens' trick.

Mertens' trick. Suppose that the central clearing house fixes $p$ as a current price vector. Checking whether a sell-order $\left(q_{\ell}, p_{\ell}^{+} / p_{c}^{+}\right)$must be (totally or, at least, partially) executed at $p$, is equivalent to solving the following programme (see Fig. 10):

$$
\max \left\{\frac{p_{\ell}^{+}}{p_{c}^{+}} \dot{x} \quad \mid \quad p \cdot \dot{x} \leq 0 \text { and } \dot{x} \geq\left(0, \ldots,-q_{\ell}, \ldots 0\right)\right\} .
$$

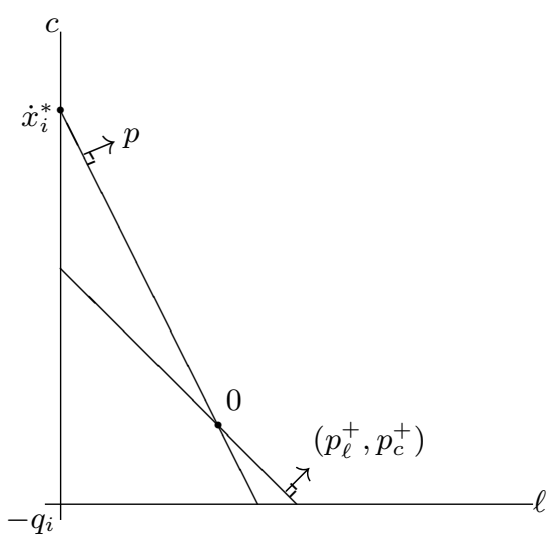

Fig. 11. Limit-price orders are fictitious linear agents. 
Here, indeed, the relative price $p_{c} / p_{\ell}$ (set by the market) is lower than the limit-price $p_{c}^{+} / p_{\ell}^{+}$, so that the order to sell $\ell$ against $c$ is entirely executed: The agent who sent this order will sell the quantity $q_{i}^{\ell}$ of good $\ell$ against $\left(p_{\ell} / p_{c}\right) q_{i}^{\ell}$ units of commodity $c$, and will end up at $\dot{x}_{i}^{*}$. But $\dot{x}_{i}^{*}$ coincides with the Walrasian demand of the fictitious agent with linear utility $v_{i}(\dot{x}):=\left(p_{c}^{+} / p_{\ell}^{+}\right) \dot{x}$ and short-sale bound $\delta_{i}:=q_{i}$. In other words, a limit-price order can be viewed as a fictitious linear "agent", whose (non-normalized) "utility" is given by the relative price (or "exchange rate", or "personal price" at which the sender of the order agrees to exchange one good for another) $p_{\ell}^{+} / p_{c}^{+}$, and whose "short-sale bound" is the offer $\left(0, \ldots, q_{\ell}, \ldots, 0\right)$.

More generally, in a $C$-commodity barter tangent market, an order is a vector of relative prices $b^{i}:=\left(p_{1}^{+}, \ldots, p_{C}^{+}\right)$and a vector of offers $e^{i}:=\left(q_{1}, \ldots, q_{C}\right)$, to be understood as follows: If the actual relative price of good $c$ against good $\ell$ verifies:

$$
\frac{p_{c}}{p_{\ell}} \geq \frac{p_{c}^{+}}{p_{\ell}^{+}}
$$

then player $i$ is ready to sell up to quantity $q_{\ell}$ of commodity $\ell$ against $c$. There is no loss of generality in restricting ourselves to sell-orders since a buying order, say of commodity $\ell$, can be replicated as an order to sell any other commodity against $\ell$. For simplicity, we assume that, within a single period $t$, a player $i$ can send a single order $\left(b^{i}, e^{i}\right)$ to the market. This also involves no loss, since this player's short-run utility is linear in $T_{z} \mathcal{E}$, so that his demand and supply correspondence can be mimicked by means of a single limit-order. ${ }^{47}$ The following examples may be helpful.

Examples. (i) If $C>2$, and $e^{i}=(0, \ldots, 1, \ldots, 0)$ ( 1 stands in the $c^{\text {th }}$ position), $b^{i}=\left(p_{1}^{+}, 0, \ldots, 0, p_{c}^{+}, 0, \ldots, 0\right)$ is a limit sell order of one unit of good $c$ in exchange for commodity 1 , with limit price $\frac{p_{c}^{+}}{p_{1}^{+}}$.

(ii) With $e^{i}$ as before, $b^{i}=\left(p_{1}^{+}, p_{2}^{+}, 0, \ldots, 0\right)$, where $\ell \notin\{1,2\}$, is a market order to sell $e_{1}^{\ell}$ in exchange for either good 1 or 2 , according to which one will yield the most value in terms of the personal relative price (i.e., "marginal utility") system $\left(p_{1}^{+}, p_{2}^{+}\right)$.

\section{ORDER BOOKS AS LINEAR ECONOMIES}

The collection of strategies played by households at time $t$ is a strategy profile, or equivalently, an order book. In the same vein as a single order is but a linear fictitious "agent", an order book can be seen as a fictitious linear "economy", $\mathcal{L}$, defined as follows:

$\triangleright\left([0,1]^{N}, \lambda_{\mid[0,1]}^{\otimes N}\right)$ is the space of "agents";

$\triangleright$ For each fictitious "agent" $i$ (i.e., for each order), his linear "utility" is given by $b^{i}:=\left(p_{c}^{+}\right)_{c}$;

$\triangleright$ His "short-sale bound" is defined as $e^{i}:=\left(q_{c}\right)_{c}$.

\footnotetext{
${ }^{47}$ By contrast, in a non-linear economy, to mimick the demand and supply correspondences of a player would require a continuum of limit-orders.
} 
Consider again the economy of Fig. 4 supra. Suppose that this two-agent linear economy is actually an order book involving for limit-price orders (one for each commodity and each fictitious agent). The unique pseudo-flow of the order book corresponding to this fictitious economy involves no-trade, whereas every point on the top horizontal segment $\left\{x \in \tau: x_{2}^{2}=0\right\}$ is Pareto-optimal. Indeed, despite the presence of positive local gainsto-trade, no-trade is the unique conceivable issue because the order corresponding to "agent" 1, having no counterpart, cannot be executed (even partially). Indeed, as in any "real" market, for an order to be executed, there must be some counterpart present on the market, and here there is none because "agent" 2 actually refuses to sell commodity 1 in exchange for 2 .

We shall make the standard assumption that every stragtegy profile induces a measurable map from the space of players into that of strategies. ${ }^{48}$ This is true, in particular, if we focus on symmetric stragtegy profiles where identical players play identical strategies. From now on, order books are therefore linear economies $\mathcal{L}=(b, e)$ defined by measurable functions $b, e:[0,1]^{N} \rightarrow \mathbb{R}_{+}^{L}$, $e$ verifying: $e_{i} \leq \delta_{i} \quad$ a.e. $i$.

Back to our dynamics, for simplicity, we impose that an order immediately disappears once it has been sent at time $t$, whether it could be executed or not. This is innocuous since, whenever the corresponding player still wants to send the same order at time $t+d t$, he simply has to re-send it. ${ }^{49}$ Given an order book $\mathcal{L}=(b, e)$, the set of flows induced by this strategy profile is defined as: $\varphi(b, e):=(P(b, e), X(b, e))$. That is, the flows induced by a strategy profile in the local game are computed by applying the definition of a flow not directly to the tangent economy $T_{z} \mathcal{E}$, but to the auxiliary linear economy $\mathcal{L}$, obtained by interpreting limit-orders as fictitious agents. ${ }^{50}$ Since the payoff of a player $i$ in the local game $G\left[T_{z} \mathcal{E}\right]$ depends only upon his (infinitesimal) allocation $\dot{x}_{i}$, and not upon prices, the indeterminacy of prices is not detrimental to the definition of the strategic flow function $f$. The key observation is that, in a perfectly competitive environment where each player is negligible with respect to others, hence cannot influence prices, no player has any incentive to manipulate his true short-run characteristics $\left(v_{i}, \delta_{i}\right)$. This turns out to hold in the more general set-up with money, to which we now turn.

\subsection{The monetary local game}

Given some stragegy profile at time $t$, the following happens:

1) At time $t_{\alpha}$ : the quantity, $\tilde{m}^{i}(t) \leq M(t)$, of Euros is borrowed by player $i$ at $t_{\alpha}$.

\footnotetext{
${ }^{48}$ See Dubey \& Shapley (1994) on this topic.

${ }^{49}$ In other words, inexecuted orders are not stored in some order book. This restriction enables to get rid of practical (and strategic) problems related to the time-to-execution of orders that are not immediately executed. Indeed, it is shown in Lo et al. [2002] that execution-time is very sensitive to the limit-price, so that in markets where orders are stored, submitting a limit-order implies a trade-off between the advantage of obtaining a fixed-price (by contrast with market orders) and the disadvantage of an unknown order execution time.

${ }^{50}$ Lemma 4 below will prove, however, that in our perfectly competitive set-up the two linear economies are the same in $L_{1}$ ).
} 
2) At time $t_{\beta}$, each player sends a message to the central clearing house, consisting of

$-p^{+i}(t):=\left(p_{1}^{+i}(t), \ldots, p_{C}^{+i}(t)\right):=$ limit-price at which $i$ is ready to sell his offer. (In order to allow comparisons, we require each such limit-price to be normalized in the unit sphere, i.e., ||$p^{+i}(t) \mid=1$.)

$-q^{i}(t):=\left(q_{1}^{i}(t), \ldots, q_{C}^{i}(t), q_{m}^{i}(t)\right):=$ quantity of commodities sent by $i$ against cash, with $q^{i}(t) \leq \delta^{i}(t)$

$-\tilde{m}_{i}(t)+\mu_{i}(t):=$ quantity of money spent by $i$, with $\mu_{i}(t) \leq m_{i}(t)$.

The clearing house interprets the received order book as a linear economy $\mathcal{L}(t)=(b(t), e(t))$ with $C$ commodities and performs trades according to Mertens'algorithm, calculating prices according to our two restrictions.

3) At time $t_{\gamma}$, each player $i$ repays $\tilde{m}_{i}(t)(1+r(t))$ to the Bank, with $r(t)$ given by

The key observation is then given by the next result, whose easy proof is left to the reader - recall, indeed, that, every player being negligible, his strategy cannot affect macro-variables such as prices; hence everything goes as if everybody would take prices as given). ${ }^{51}$

Lemma 4.- In any tangent market $T_{z(t)} \mathcal{E}$, "truth-telling", i.e.,

$$
b_{i}(t)=\lambda(t) \nabla u_{i}\left(x_{i}(t)\right) \quad \text { for some } \lambda(t)>0 \text { and } e_{i}(t)=\delta_{i}(t) \quad \text { a.e. } i,
$$

is a dominant-strategy equilibrium in $G\left[T_{z(t)} \mathcal{E}\right]$.

\section{Concluding remarks}

We end with a few final remarks.

a) Although outside money plays a crucial rôle in our model, its presence remains questionable from an economic point of view. In subsequent work, we plan to explore the impact of allowing for a certain amount of default along trade curves, taking inspiration from Tsomocos (2003). Default, indeed, is known to be able to play a rôle analogous to outside money in the analysis of money in a general equilibrium setting - cf. Espinosa, Goodhart \& Tsomocos (2009). The idea would be to see whether inside money and

\footnotetext{
${ }^{51}$ The picture would be completely different in the presence of finitely many players. Then, of course, the aggregate order book, $\mathcal{L}$, resulting from, say, a Nash equilibrium, could be quite different from the underlying marginal economy $T_{z} \mathcal{E}$. See Weyers [2003] for a first study of the strategic market game associated to the limit-price mechanism in the imperfectly competitive case.
} 
default (without outside money) would induce the same kind of results as in this paper or would capture different economic properties. ${ }^{52}$ On the other hand, allowing for default will enable us to further weaken the concept of "locally rational" expectations - which, as already mentioned, imply $p \cdot \dot{x}_{i}=0$, for every household $i$, in this paper. Finally, it will open the door for a dynamic study of the systemic risk of collective crashes.

b) Everyday experience on the interbank market suggests that (at least in Europe) this is a rather highly imperfectly competitive market, where a few "big" atomic players interact strategically. Thus, this first study calls for a parallel analysis within an imperfectly competitive framework. This implies studying Mertens' limit-price mechanism with finitely many players. A first step in this direction has been made by Weyers (2003). We also plan to consider the Central Bank itself as a player in order to study within the present framework the most debated issue of its credibility.

c) A quantitative analysis of the long-run impact of money will be performed latler on, taking advantage of the generic global nominal uniqueness of trade curves in our dynamics, and of the fact that this dynamics is numerically computable (as can be deduced from the proof of Theorem 2, see the Appendix).

\section{References}

[1] Aubin, J.-P. \& A. Cellina (1984) Differential Inclusions, Springer-Verlag, Berlin.

[2] Bloise, G. \& H. M. Polemarchakis (2006) "Introduction to the Symposium", Economic Theory, 27:1, 1-23.

[3] Bonnisseau, J.-M., M. Florig, A. Jofre (2001) "Differentiability of Equilibria for Linear Exchange Economies" J. Optim. Theory Appl. 109, no. 2, pp. 265-288.

[4] Bottazzi, J.-M. (1994) "Accessibility of Pareto Optima by Walrasian Exchange Processes", Journ. of Math. Economics, 23, 585-603.

[5] Champsaur, P., and B. Cornet (1990) "Walrasian Exchange Processes", in: Gabszewicz, J.J., Richard, J.-F., Wolsey, L.A. (eds.) Economic Decision Making: Games, Econometrics and Optimizaiton. Amsterdam: Elsevier.

[6] Champsaur, P., J. H. Drèze \& Cl. Henry (1977) "Stability Theorems with Economic Applications" Econometrica, 45(2), 273-94.

[7] Clower, R. (1967) "A Reconsideration for the Microeconomic Foundations of Monetary Theory", Western Economic Journal, 6, 1-8.

[8] Cornet, B. (1989) "Linear Exchange Economies", Cahier Eco-Math, Université de Paris 1.

[9] Coste, M. (2000) An Introduction to O-minimal geometry, Università di Pisa, lecture notes.

[10] Drèze, J. \& H. Polemarchakis (1999) "Money and Monetary Policy in General Equilibrium", in L.-A. Gérard-Varet, A. P. Kirman \& M. Ruggiero (eds.), Economics, the Next Ten Years, Oxford, Oxford University Press.

[11] (2000) "Intertemporal General Equilibrium and Monetary Theory", in A. Leijonhufvud (ed.), Monetary Theory as a Basis for Monetary Policy, Macmillan.

\footnotetext{
${ }^{52}$ Default and different lending and deposit rates as in Goodhart, Sunirand \& Tsomocos (2006) also allow for analyzing credit spreads.
} 
$[12]$ (2001) "Monetary Equilibrium", in G. Debreu, W. Neuefeind \& W. Trockel (eds.) Economics Essays - A Festschrift for Werner Hildenbrand, Springer.

[13] Dubey P. \& J. Geanakoplos (1992) "The Value of Money in a Finite Horizon Economy: A Role for Banks", in Dasgupta, P., Gale, D. et alii (eds), Economic Analysis of Market and Games, MIT Press, Cambridge, 407-444.

[14] (2003a) "Inside and Outside Money, gains-to-trade and IS-LM", Economic Theory 21, 347-397.

[15] (2003b) "Monetary Equilibrium with Missing Markets", Journ. of Math. Economics, 39, 585-613.

[16] (2006) "Determinacy with Nominal Assets and Outside Money", Economic Theory, 27:1, 79-106.

[17] Dubey, P. \& L. S. Shapley (1994) "Non-cooperative Exchange with a Continuum of Traders: two Models", Journ. of Math. Econ., 23(3), 253-293.

[18] Dubey, P. \& M. Shubik (1978) "The Non-cooperative Equilibria of a Closed Trading Economy with Market Supply and Bidding Strategies", Journ. of Economic Theory, 17, 1-20.

[19] Espinoza, R., Ch. Goodhart \& D. Tsomocos (2009) 'State prices, liquidity, and default" Economic Theory, 39(2), 177-194.

[20] Filippov, A.I. (1988) Differential Equations with a Discontinuous Right-hand Side, Kluwer Academic Publisher.

[21] Florig, M. (2001) 'Hierarchic Competitive Equilibria'", Journ. of Math. Econ., 35, 515-546.

[22] Giraud, G. (2003) "Strategic Market Games: an Introduction", Journ. of Math. Econ., 39, $355-375$.

[23] (2004) "The Limit-price Exchange Process", Cahiers de la MSE b04118, Université Panthéon-Sorbonne (Paris 1).

[24] (2009) "From Non-tâtonnement to Monetary Dynamics within General Equilibrium Theory", forthcoming in: J.-S. Lenfant (ed) General Equilibrium as Knowledge. From Walras onwards.

[25] Giraud, G. \& D. Tsomocos (2004) "Global Uniqueness and Money Non-Neutrality in a Walrasian Dynamics without Rational Expectations", OFRC fe-15 , University of Oxford, Saïd Business School.

[26] Goodhart, C.A.E., P. Sunirand \& D.P. Tsomocos (2006) "A Model to Analyse Financial Fragility", Economic Theory, 27, 107-142.

[27] Grandmont, J.-M. (2007) "Temporary Equilibrium", in The New Palgrave, Lawrence Blume and Steve Durlaub (Eds.), Palgrave Mc Millan.

[28] Grandmont, J.-M. \& Y. Younès (1972) "On the Role of Money and the Existence of Monetary Equilibrium", Review of Economic Studies 39, 355-372.

[29] Gray, L. \& J. Geanakoplos (1991), "When Seeing Further is not Seeing Better", Bulletin of the Santa Fe Institute 6(2): 1-6.

[30] Gurley, J.G. \& E.S. Shaw (1960) Money in a Theory of Finance, Washington, DC: Brookings

[31] Hahn, F.-H. (1965) "On Some Problems of Proving the Existence of an Equilibrium in a Monetary Economy", in Hahn, F.H. \& F.R.P. Brechling (eds) The Theory of Interest Rates, MacMillan, New-York. 
[32] Kirman, A. P. (1992) "Whom or What Does the Representative Individual Represent?" Journ. Econ. Perspectives, 8 (2), pp. 11736.

[33] Lucas, R. (1972) "Expectations and the Neutrality of Money", Journ. of Economic Theory $4,103-124$.

[34] Mas-Colell, A. (1985) The Theory of General Economic Equilibrium: A Differentiable Approach, Econometric Society Monograph, Cambridge University Press, Cambridge.

[35] Mertens, J.-F. (2003) "The limit-price mechanism", Journ. of Math. Economics, 39, 433528.

[36] Sahi, S. \& S. Yao (1989) "The Non-cooperative Equilibria of a Trading Economy with Complete Markets and Consistent Prices", Journ. of Math. Econ., 18, 325-346.

[37] Shapley, L. S. \& M. Shubik (1977) "Trading using one Commodity as a Means of Payment", Journ. of Political Economy 85(5), 937-968.

[38] Shubik, M. \& D.P. Tsomocos (1992) "A Strategic Market Game with a Mutual Bank with Fractional Reserves and Redemption in Gold", Journal of Economics, 55(2), 123-150.

[39] Shubik, M. \& C. Wilson (1977) "The Optimal Bankuptcy Rule in a Trading Economy Using Fiat Money", Journal of Economics, 37, 337-354.

[40] Smale, S. (1976a) "Dynamics in General Equilibrium Theory", American Economic Review, $66,288-294$.

[41] (1976b) "Exchange Processes with Price Adjustment", Journ. of Math. Econ., 3, 211-226.

[42] _ (1977) "Some Dynamical Questions in Mathematical Economics", Colloques Internationaux du Centre National de la Recherche Scientifique, N 259: Systèmes Dynamiques et Modèles Économiques.

[43] Tsomocos, D. (2003) "Equilibrium Analysis, Banking and Financial Instability", Journ. of Math. Econ., 39, 619-655.

[44] Tsomocos, D. (2008) "Generic Determinacy and Money Non-Neutrality of International Monetary Equilibria", Journ. of Math. Economics, 44 (7-8), 866-887.

[45] Weyers, S. (2003) "A Strategic Market Game with Limit Prices", Journ. of Math. Economics, 39, 529-558.

\section{Appendix}

This section contains mathematical proofs of results not proven in the main text.

In order to prove Theorem 1, we need to recall Filippov's solution for differential equations with a discontinuous right-hand side. Let

$$
\dot{x}(t)=f(x(t)),
$$

where $f: \mathbb{R}^{m} \leftrightarrow \mathbb{R}^{m}$ is a possibly discontinuous vector field. 
Definition. A Filippov solution of (32), is an absolutely continuous trajectory $\phi:[a, b) \rightarrow \mathbb{R}^{m}$ such that, for a.e. $t \in[a, b)$,

$$
\left.\dot{\phi}(t) \in G_{f}(\phi(t)):=\cap_{\varepsilon>0} \cap_{A \in \mathcal{N}} \overline{\operatorname{co}}\{y \mid d(y, f(\phi(t))))<\varepsilon, y \notin A\right\} .
$$

where $\mathcal{N}:=$ family of sets $A \subset \mathbb{R}^{m}$ of (Lebesgue) measure zero.

In words, a path $\phi$ is a solution of (32) if it is absolutely continuous and if, for almost all $t \in[a, b)$, and for arbitrary $\varepsilon>0$, the vector $\frac{d}{d t} \phi(t)$ belongs to the smallest convex closed set containing all the values of the sets $f(y)$, when $y$ ranges over almost all of the $\varepsilon$-neighborhoods of $x$, i.e., the entire neighborhood except possibly for a set of Lebesgue measure zero.

\section{Proof of Theorem 1.}

In order to apply Filippov's theory, we need to slightly perturb the dynamics defined by (24). Indeed, as we know from the remark just after Lemma 2 that, for $\dot{x}(t)=0$, $P\left(T_{z(t)} \mathcal{E}\right)$ does not reduce to a singleton. Thus, for all rest-points of the dynamics, we can replace $P\left(T_{z(t)} \mathcal{E}\right)$ by an arbitrary selection $p_{z(t)} \in P\left(T_{z(t)} \mathcal{E}\right)$. Similarly, for every $t$ such that $\bar{\mu}(t)=0$ and $M(t)>0$, we know that the resulting pseudo-flow is indeterminate because it is an "optimal allocation" (Mertens (2003)) where prices are only defined up to a normalization factor $\lambda$, so that $P\left(T_{z} \mathcal{E}\right)$ is an open half-line. To get rid of this, and in order to recover a (possibly discontinuous) ordinary differential equation on which Filippovs' theory applies, it suffices, again, to adopt a conventional normalization rule in such degenerate cases - for instance, one could normalize prices into the unit sphere. Let us denote by $\tilde{P}\left(T_{z(t)} \mathcal{E}\right)$ the resulting (discontinuous) price function. We can now complete our definition of trade curves by replacing the so far unspecified word "solution" with Filippov solution in the following rewriting of (24):

$$
(p(t), \dot{x}(t))=f(z(t)):=\left(\tilde{P} x\left(T_{z(t)} \mathcal{E}\right), X\left(T_{z(t)} \mathcal{E}\right)\right) .
$$

Now, Filippov's set-valued map $G_{f}$ associated to the vector field $f$ is easily seen to be upper semi-continuous, non-empty, convex-, and compact-valued, and locally bounded. In particular, local boundedness comes from the fact that, $\dot{x}(t)$ being feasible, it is uniformly bounded, while the cash-in-advance constraints (10) and the continuity of the monetary policy $M(\cdot)$ imply the local boundedness of quasi-flow prices. Thus the Theorem is a consequence of a classical existence result for differential inclusions, e.g., in Aubin \& Cellina (1984 chap. 2)

Remark. Of course, we do not use the kind of trick applied here in order to recover a vector field for Theorem 2 since, there, what is at stake is precisely the nominal determinacy of prices, while the preceding proof, being only concerned with existence, allows itself to artificially circumvent the price indeterminacy.

In order to prove Theorem 2, we need to recall some technical background material borrowed from algebraic geometry. The appropriate mathematical set-up for introducing 
the class of semi-analytic economies is that of 0-minimal Tarski systems (see Coste (2000)). However, we content ourselves, here, with the more modest class of semianalytic sets - which is quite sufficient for our purposes.

A subset $X \subset \mathbb{R}^{n}$ is semi-analytic if, for each $y \in \mathbb{R}^{n}$, there is an open neighborhood $U$ of $y$ such that $U \cap X$ is the finite union of sets defined by real analytic equalities and inequalities. Formally, $U \cap X$ is the finite disjoint union of sets of the form $\left\{x \mid f_{i}(x)=\right.$ $\left.\alpha_{i}, g_{j}(x)>\beta_{j}, 1 \leq i \leq M, 1 \leq j \leq N\right\}$, where $f_{i}$ and $g_{j}: \mathbb{R}^{n} \rightarrow \mathbb{R}$ are real-analytic functions.

A function $f: \mathbb{R}^{n} \rightarrow \mathbb{R}^{m}$ is said to be semi-analytic whenever its graph, Graph $f \subset \mathbb{R}^{n+m}$, is so.

A subclass of the category of semi-analytic sets is provided by the semi-algebraic sets, i.e., those that are obtained from the definition of a semi-analytic set after having replaced "real-analytic" by "polynomial". Many transcendental functions are semianalytic but not semi-algebraic: so are the restrictions of the exponential function, the logarithm and the trigonometric functions to compact subsets of their domains. Compositions, algebraic combinations, and derivatives of semi-analytic functions are semianalytic, but indefinite integrals are not. Neither are the exponential, the logarithm and the trigonometric functions on their entire domains.

Proof of Theorem 2.

We first claim that, given $\omega \gg 0$, the set of preferences representable by a semianalytic utility is dense in the space of $\mathcal{C}^{2}$ utilities satisfying the standard smoothness assumptions. This follows from the standard proof showing that smooth preferences are dense in the space of $\mathcal{C}^{2}$ utilities (see, e.g., Mas-Colell (1985, Prop. 2.8.1. p. 90)) by keeping track of the fact that every object involved in the construction of the approximating sequence of smooth preferences must be semi-analytic. For this, one simply needs to observe that:

(i) for any integer $n>0$, a $\mathcal{C}^{\infty}$-density function $\xi_{n}: \mathbb{R}^{\ell} \rightarrow \mathbb{R}$ with support containing the origin and radius $\leq \frac{1}{n}$ can be constructed so as to be semi-analytic;

(ii) If $v, \xi_{n}: \mathbb{R}^{\ell} \rightarrow \mathbb{R}$ are finitely subanalytic, so is the restriction of the convolution

$$
u_{n}^{\prime}(x):=\int_{\mathbb{R}} v(x-z) \xi_{n}(z) d z
$$

to the compact $\tau$. (Notice that the support of $z$ in the integral is bounded.)

Stone-Weierstrass theorem then enables us to uniformly approximate the monetary policy $M(\cdot)$ as well as expectations maps, $\delta_{i}(\cdot)$ and $\mu_{i}(\cdot)$ (every $i$ ), by a polynominal, hence by a finitely sub-analytic function. Observe that approximating utilities being semi-analytic and differentiable, so is their differential. It suffices, indeed, to express the differential as a limit of variation rates, and to apply Tarski-Seidenberg theorem. Thus, the graph of the map $z \mapsto T_{z} \mathcal{E}$ can be expressed by a finite number of polynomial equalities and inequalities, hence it is itself semi-analytic (in fact, semi-algebraic). But the map that associates to each tangent economy its monetary flow is semi-analytic as well, by the same argument. Thus (cf. Coste (2000, Lemma 6.8, p. 71)), there exists an open, semi-analytic subset $\mathcal{R}$ of $\mathbb{R} \times \mathcal{M}^{*}$ such that the restriction, $f_{\mid \mathcal{R}}$, of the 
vector field $f$ defined by $(34)$ is real-analytic (hence $\left.\mathcal{C}^{\infty}\right)$ and $\operatorname{dim}\left(\left(\mathbb{R} \times \mathcal{M}^{*}\right) \backslash \mathcal{R}\right)<\operatorname{dim}$ $\left(\mathbb{R} \times \mathcal{M}^{*}\right)=C(N-1)+(N+1)(C+2)+1$. Obviously, $\mathcal{R}$ is dense in $\mathbb{R} \times \mathcal{M}^{*}$. It suffices to define $\mathcal{C}:=\left(\mathbb{R} \times \mathcal{M}^{*}\right) \backslash \mathcal{R}$.

The set $\mathcal{C}$ of critical economies being semi-analytic, it is the finite, disjoint union of smooth submanifolds, all of them of dimension less than $C(N-1)+N(C+2)+2$.

\section{Remarks}

(a) At first glance, no transversality argument seems to appear in the proof of the generic global uniqueness and smoothness of trajectories in our argument. Actually, transversality is "hidden" behind the property that every semi-analytic set is locally trivial. This point plays the role, in algebraic geometry, of Sard's theorem, and is at the heart of the fact that a finitely subanalytic map is almost everywhere real-analytic.

(b) The discussion following Theorem 2 is an informal development of the following. Consider a trade curve $x(\cdot)$ crossing a $\mathcal{C}^{1}$ hypersurface $S$ at some point $x$ at time, say, $T$. Let the interior of the feasible set $\tau^{*}$ be separated by $S$ into domains $G^{-}$and $G^{+}$. The partial derivatives $\frac{\partial \varphi}{\partial x_{k}}, k=1, \ldots, C(N-1)$ are continuous in domains $G^{+}$and $G^{-}$ up to the boundary. Let $\varphi^{-}(x)$ and $\varphi^{+}(x)$ be the limiting values of the function $f$ at the point $x \in S$, from the domains $G^{-}$and $G^{+}$respectively. Let

$$
h(x):=\varphi^{+}(x)-\varphi^{-}(x),
$$

be the discontinuity vector at $x$ of our vector field. Finally, let $\varphi_{N}^{-}, \varphi_{N}^{+}, h_{N}$ be the (orthogonal) projections of the vectors $\varphi^{-}, \varphi^{+}, h$ onto the normal line to $S$ directed from $G^{-}$to $G^{+}$at the point $x$. Within the domains $G^{-}$and $G^{+}$, right and left uniqueness of solution to (24) holds true (Cauchy-Lipschitz theorem). All we therefore need is to study what happens in a neighborhood of the hypersurface $S$. The following Proposition summarizes the various situations we may encounter:

Proposition.- $\left(\right.$ Filippov $\left.^{53}\right)$ If $S$ is $\mathcal{C}^{2}$ and the function $h(x)=f^{+}(x)-$ $f^{-}(x)$ is $\mathcal{C}^{1}$ at each point $x \in S$, if, moreover, at least one of the inequalities $f_{N}^{-}>0$ or $f_{N}^{+}<0$ (possibly different inequalities for different $x$ ) holds, the right uniqueness for (24) occurs for $a<t<b$ in $G$.

\section{Proof of Proposition 3.}

When $\bar{\mu}>0$ for every $i$, the "real" and the "monetary" parts, $\left((\lambda(t) p(t))_{\lambda(t)>0}, \dot{x}(t)\right)$ and $(\lambda(t), \tilde{m}(t))$, of a monetary flow can be separated by excluding from consideration those traders $i$ for whom $\bar{\mu}_{i}=0$ (who do not trade anyway). Moreover, every pseudoflow is of first-order so that, whithin the linear economy restricted to traders endowed with ouside money, the pair of price ratios and net trades $\left.(\lambda p(t))_{\lambda>0}, \dot{x}(t)\right)$ is solely

\footnotetext{
${ }^{53}$ See Filippov (1968), Lemma 2 and Corollary 1 (p. 107), Corollary 2 and Lemma 3 (p. 108) and Theorem 2 (p. 110).
} 
defined by: $\dot{x}(t)$ is feasible, $\dot{x}_{i}(t) \geq-\delta_{i}(t)$ and $p(t) \cdot \dot{x}_{i}(t)=0$ for every $i$. In other words, $\left.(\lambda p(t))_{\lambda>0}, \dot{x}(t)\right)$ is but a Walrasian equilibrium (in net trades) of the restriction of $T_{x(t)} \mathcal{E}$ to people with endowed money. The gross-substitutability property (25) then follows from Bonnisseau, Florig \& Jofré (2001, corollary 4.2). When $\bar{\mu}=0$, every monetary flow is either $(M>0)$ a Walras equilibrium of the whole economy $T_{z} \mathcal{E}$, or autarky $(M=0)$ : in both cases, the result follows again.

\section{Proof of Proposition 4.}

(i) Remark, first, that, since $\nabla u_{i}\left(x_{i}\right) \gg 0$, for every $i$, every pseudo-flow is of firstorder. Suppose that $\gamma(x)>r \geq 0$, and that nevertheless the monetary flow of $T_{z} \mathcal{E}$ involves no-trade. Then, for every household $i$, the cash-in-advance constraint $p \cdot \dot{x}_{i}^{+} \leq$ $\mu_{i}\left(\frac{1+r}{r}\right)$ is trivially satisfied, whatever being people's initial endowment in money as well as the factor $\lambda>0$ chosen by the clearing house in order to fix the price level $p^{\lambda}$. Imagine therefore that the clearing house commits to set $\lambda>0$ and let $\lambda \rightarrow+\infty$. Since, at a monetary flow, $p \gg 0$ by definition, this means that $p^{\lambda} \rightarrow+\infty$ as well. As a consequence, the purchasing power of the endowed money $m_{i}$ goes to zero and may be ignored. At the limit, the trading opportunity for any household is to purchase goods solely out of the borrowed money and to pay back the loan $n_{i} M$ at the interest rate $r$, out of his sales revenues - conducting all infinitesimal trades at $p^{*}$. To be more precise, consider the limiting price ratios given by $p^{*}$, where:

$$
p_{c}^{*}:=\lim _{\lambda \rightarrow \infty} \frac{p_{c}^{\lambda}}{\sum_{k} p_{k}^{\lambda}}, \quad \text { every } c,
$$

and let us denote by $B_{i}\left(p^{\lambda}, \mu^{\lambda}, r\right)$, the budget set of agent $i$ defined by those infinitesimal trades $\dot{x}_{i} \geq-\delta_{i}$ such that the cash-in-advance constraint (10) and the no-default constraint (11) are satisfied. As shown by Lemma 3, this is equivalent to:

$$
p^{\lambda} \cdot \dot{x}_{i}^{+}+\frac{1}{1+r} p^{\lambda} \cdot \dot{x}_{i}^{-} \leq \mu_{i} .
$$

The budget set being therefore homogeneous with respect to $(p, \mu)$, one has:

$$
B_{i}\left(p^{\lambda}, \mu^{\lambda}, r\right)=B_{i}\left(\frac{p^{\lambda}}{\left\|p^{\lambda}\right\|_{\ell_{1}}}, \frac{\mu}{\left\|p^{\lambda}\right\|_{\ell_{1}}}, r\right)
$$

where $\left|p^{\lambda}\right|_{\ell_{1}}:=\sum_{k} p_{k}^{\lambda}$. As $\lambda \rightarrow \infty$, we have the set convergence of this budget set towards $B_{i}\left(p^{*}, 0, r\right)$. If, now, $\dot{x}_{i}$ is $v_{i}$-optimal in $B_{i}\left(\frac{p^{\lambda}}{\left|p^{\lambda}\right| \ell_{1}}, \frac{\mu}{\left|p^{\lambda}\right| \ell_{1}}, r\right)$, its limit, $\dot{x}_{i} \rightarrow 0$, must be $v_{i}$-optimal in $B_{i}\left(p^{*}, 0, r\right)$. In the same way as in Theorem 2 of Dubey \& Geanakoplos (2003a), this is tantamount to performing standard Walrasian trades at $p^{*}$ but consuming only the fraction $1 /(1+r)$ of purchases. In turn, a change of variable shows that this may be viewed as performing the whole Walrasian net trades via modified utilities $v_{i}^{r}$ defined as follows:

$$
v_{i}^{r}\left(\dot{x}_{i}\right):=v_{i}\left(\dot{x}_{i}(r)\right),
$$


where $\dot{x}_{i}(r)$ is defined as in (26). Thus, no-trade is a Walras allocation for $\left(v_{i}^{r}\right)_{i}$ at prices $p^{*}$, and must be Pareto-optimal wrt $\left(v_{i}^{r}\right)_{i}$. Since $r<\gamma(x), 0$ is also Pareto optimal with respect to $\left(v_{i}^{\gamma(x)}\right)_{i}$. But we know from Lemma 2 in Dubey \& Geanakoplos (2003a) that there are no local gains to $\gamma$-diminished trades in $T_{z} \mathcal{E}$ if, and only if, the (concave but non-linear) economy $\left(v_{i}^{\gamma}\right)_{i}$ has a no-trade Walras equilibrium. This contradicts the gains-to-trade hypothesis $\gamma(x)>0$. So, no-trade cannot be a monetary flow of $T_{z} \mathcal{E}$.

(ii) is a direct consequence of Theorem 6 in Dubey \& Geanakoplos (2003a). There, it is proven that, under the stated condition, no individual can have effective trades since she could then be able to improve her short-run welfare by slightly perturbing her trades. The same argument shows, here, that the unique monetary flow must be no-trade. Details are left to the reader.

\section{Proof of Theorem 3.}

(i) Define the Lyapounov function $\mathcal{V}: \tau \rightarrow \mathbb{R}$ by:

$$
\mathcal{V}(x)=\sum_{i} u_{i}\left(x_{i}\right)
$$

For every trade curve solving (24), one has for a.e. $t>0$ :

$$
\frac{d}{d t} \mathcal{V}(x(t))=\sum_{i} \nabla u_{i}\left(x_{i}(t)\right) \cdot \dot{x}_{i}(t) .
$$

It follows from Proposition 4 that $\frac{d}{d t} \mathcal{V}(x(t))=0 \Longleftrightarrow x(t) \in \theta$, otherwise, $\frac{d}{d t} \mathcal{V}(x(t))>0$. Indeed, if $x(t)$ is locally Pareto-optimal, then Proposition 4 implies that no-trade is the unique monetary flow of the tangent market attached to $x$, so that $\frac{d}{d t} \mathcal{V}(x(t))=0$. Conversely, if $\frac{d}{d t} \mathcal{V}(x(t))=0$, then $\nabla u_{i}(x(t)) \cdot \dot{x}_{i}=0$ for every type of household $i$. Let $(p(t), \tilde{m}(t), \dot{x}(t))$ be a monetary flow of $T_{z(t)} \mathcal{E}$, the tangent market corresponding to $x(t)$. Under the standard smoothness assumptions, since $\bar{\mu}(t)>0$ and $\delta_{i}(t)>0$ for every $i$, every pseudo-flow is of first-order so that the pair of price ratios and net trades $\left.(\lambda p(t))_{\lambda>0}, \dot{x}(t)\right)$ is solely defined by: $\dot{x}(t)$ is feasible, $\dot{x}_{i}(t) \geq-\delta_{i}(t)$ and $p(t) \cdot \dot{x}_{i}(t)=0$ for every $i$. One therefore gets from the duality theorem on linear programming, for every $i$, that $\nabla u_{i}\left(x_{i}(t)\right) \cdot \dot{x}_{i}(t)=0$ for every $i \Rightarrow \dot{x}(t)=0$ (see, e.g., Champsaur \& Cornet 1990, Lemma 2 for details).

Now, it follows from the properties of the Lyapounov function $\mathcal{V}(\cdot)$ and from Champsaur, Drèze \& Henry (1977), that every limit-point $x^{*}$ of a solution of (34) belongs to $\theta$. (That every solution admits at least one limit-point follows from the compactness of $\tau$.)

(ii) Proposition 4 and the stated condition imply that, for a time set of positive length, $0 \in G_{f}(x(t))$. Therefore, $x(t)$ is a rest-point of the Filippov's solution of (34).

(iii) Take $x \in \theta$, and some neighborhood $V$ of $x$ in $\tau$. Since $\mathcal{V}$ is continuous, let $\underline{v}>0$ be the maximum of $\mathcal{V}$ over the frontier $\bar{V} \backslash V$. Consider, now, the subset $U:=\{y \in$ 
$\tau \mid \mathcal{V}(y)=\underline{v}+\varepsilon\} \cap V$. Clearly, $U$ is included in $V$, contains $x$, and if a solution starts in $U$, it cannot escape from $U$. Hence, $x$ is locally stable.

(iv) That the trade curves $x_{k}(\cdot)$ are identical on $\left[t_{a}, t_{b}\right]$ is a consequence of the fact that, as already observed in the proof of Proposition 3 above, when $\bar{\mu}>0$ and $\delta_{i} \gg>$, the "real" and the "monetary" parts, $\left((\lambda(t) p(t))_{\lambda(t)>0}, \dot{x}(t)\right)$ and $(\lambda(t), \tilde{m}(t))$ can be separated. The real part is independent from $M(t)$ : Only the price normalization factor $\lambda(t)>0$ and the amounts of borrowed money $\tilde{m}_{i}(t)$ depend upon $M(t)$. 